\title{
Missing water in Class I protostellar disks ${ }^{\star}$
}

\author{
D. Harsono ${ }^{1, \star \star}$, M. V. Persson ${ }^{2}$, A. Ramos ${ }^{3}$, N. M. Murillo ${ }^{1}$, L. T. Maud ${ }^{1,4}$, M. R. Hogerheijde ${ }^{1,5}$, A. D. Bosman ${ }^{1}$, \\ L. E. Kristensen ${ }^{6}$, J. K. Jørgensen ${ }^{6}$, E. A. Bergin ${ }^{7}$, R. Visser ${ }^{4}$, J. C. Mottram ${ }^{8}$, and E. F. van Dishoeck ${ }^{1,9}$ \\ ${ }^{1}$ Leiden Observatory, Leiden University, Niels Bohrweg 2, 2300 RA Leiden, The Netherlands \\ e-mail: harsono@strw. leidenuniv.nl \\ 2 Department of Space, Earth and Environment, Chalmers University of Technology, Onsala Space Observatory, 43992 Onsala, \\ Sweden \\ ${ }^{3}$ Department of Astronomy, University of Texas, Austin, TX, USA \\ ${ }^{4}$ European Southern Observatory, Karl-Schwarzschild-Straße 2, 85748 Garching, Germany \\ 5 Anton Pannekoek Institute for Astronomy, University of Amsterdam, Science Park 904, 1098 XH Amsterdam, The Netherlands \\ ${ }^{6}$ Niels Bohr Institute \& Centre for Star and Planet Formation, University of Copenhagen, Øster Voldgade 5-7, 1350 Copenhagen K, \\ Denmark \\ ${ }^{7}$ Department of Astronomy, The University of Michigan, 500 Church St., 830 Dennison Bldg., Ann Arbor, MI 48109, USA \\ ${ }^{8}$ Max Planck Institute for Astronomy, Königstuhl 17, 69117 Heidelberg, Germany \\ ${ }^{9}$ Max Planck Institut für Extraterrestrische Physik, Giessenbachstrasse 1, 85748 Garching, Germany
}

Received 31 May 2019 / Accepted 24 February 2020

\section{ABSTRACT}

Context. Water is a key volatile that provides insight into the initial stages of planet formation. The low water abundances inferred from water observations toward low-mass protostellar objects may point to a rapid locking of water as ice by large dust grains during star and planet formation. However, little is known about the water vapor abundance in newly formed planet-forming disks.

Aims. We aim to determine the water abundance in embedded Keplerian disks through spatially-resolved observations of $\mathrm{H}_{2}^{18} \mathrm{O}$ lines to understand the evolution of water during star and planet formation.

Methods. We present $\mathrm{H}_{2}^{18} \mathrm{O}$ line observations with ALMA and NOEMA millimeter interferometers toward five young stellar objects. NOEMA observed the $3_{1,3}-2_{2,0}$ line $\left(E_{\text {up }} / k_{\mathrm{B}}=203.7 \mathrm{~K}\right)$ while ALMA targeted the $4_{1,4}-3_{2,1}$ line $\left(E_{\text {up }} / k_{\mathrm{B}}=322.0 \mathrm{~K}\right)$. Water column densities were derived considering optically thin and thermalized emission. Our observations were sensitive to the emission from the known Keplerian disks around three out of the five Class I objects in the sample.

Results. No $\mathrm{H}_{2}^{18} \mathrm{O}$ emission is detected toward any of our five Class I disks. We report upper limits to the integrated line intensities. The inferred water column densities in Class I disks are $N_{\mathrm{H}_{2}^{18} \mathrm{O}}<10^{15} \mathrm{~cm}^{-2}$ on 100 au scales, which include both the disk and envelope. The upper limits imply a disk-averaged water abundance of $\lesssim 10^{-6}$ with respect to $\mathrm{H}_{2}$ for Class I objects. After taking the physical structure of the disk into account, the upper limit to the water abundance averaged over the inner warm disk with $T>100 \mathrm{~K}$ is between $\sim 10^{-7}$ and $10^{-5}$.

Conclusions. Water vapor is not abundant in warm protostellar envelopes around Class I protostars. Upper limits to the water vapor column densities in Class I disks are at least two orders of magnitude lower than values found in Class 0 disk-like structures.

Key words. stars: protostars - stars: formation - ISM: abundances - astrochemistry - protoplanetary disks

\section{Introduction}

Water is strongly connected to the emergence of life and the formation of planetary systems (Chyba \& Hand 2005; Kitadai \& Maruyama 2018). Water also plays an important physical role during star and planet formation, from acting as a gas coolant allowing clouds to collapse (e.g., Goldsmith \& Langer 1978; Neufeld et al. 1995; Karska et al. 2018) to assisting the coagulation of ice-covered grains in disks beyond the snow line (Stevenson \& Lunine 1988; Gundlach \& Blum 2015; Schoonenberg \& Ormel 2017).

Thanks to infrared and submillimeter observations over recent decades, the water abundances in gas and ice have been

\footnotetext{
* The reduced spectral cubes are only available at the CDS via anonymous ftp to cdsarc.u-strasbg. fr (130.79.128.5) or via http:// cdsarc.u-strasbg.fr/viz-bin/cat/J/A+A/636/A26

${ }^{\star \star}$ Current affiliation: EACOA Fellow, Institute of Astronomy and Astrophysics, Academia Sinica, 11F of ASMAB, AS/NTU No. 1, Sec. 4, Roosevelt Road, Taipei 10617, Taiwan.
}

measured at each of the evolutionary stages from clouds to planets (see van Dishoeck 2004; Melnick 2009; Hogerheijde et al. 2011; Kristensen et al. 2017a,b). In parallel, laboratory experiments and quantum chemical calculations have provided deep insight into the basic molecular processes considered in the astrochemical networks used to explain the observed water abundances (Burke \& Brown 2010; van Dishoeck et al. 2013; Arasa et al. 2015). One of the key stages in this evolutionary path for which information is still missing is that of disk formation (van Dishoeck et al. 2014).

Infrared observations have shown that water ice is abundant, $\sim 10^{-4}$ with respect to $\mathrm{H}_{2}$, in cold dense clouds $\left(n>10^{4} \mathrm{~cm}^{-3}\right.$, $T_{\text {dust }} \sim 10 \mathrm{~K}$, Whittet et al. 1988; Smith et al. 1989; Gibb et al. 2004; Boogert et al. 2015), locking up much of the available oxygen. During the collapse of a dense core, water ice is preserved until the inner envelope $(<1000 \mathrm{au})$ heats up: once temperatures above $100 \mathrm{~K}$ are reached close to the protostar, water ice starts to sublimate. Water vapor is also rapidly produced in high abundances $\left(\geq 10^{-5}\right)$ in warm high-velocity shocked gas 
associated with outflows, where it is prominently seen in its bright far-infrared and submillimeter lines with the ISO, SWAS, ODIN, and Herschel missions, which probe the envelope scales (>1000 au, e.g., Nisini et al. 2002; Snell et al. 2000; Olofsson et al. 2003; Kristensen et al. 2012; Mottram et al. 2014, 2017, and see Bergin \& van Dishoeck 2012 for a review). Shocked water is, however, largely lost to space and does not contribute to the inventory of planet-forming disks (Visser et al. 2009).

Determining the water abundance in planet-forming disks ( $\sim 100$ au scales) has been remarkably difficult. Herschel-HIFI targeted the cold gaseous water in protoplanetary disks (Class II) with little success. Cold water vapor detections using the groundstate $\mathrm{H}_{2} \mathrm{O}$ line have been reported for just two disks (Hogerheijde et al. 2011; Salinas et al. 2016) and stringent upper limits for a dozen other sources at an order of magnitude lower than expected (Bergin et al. 2010; Du et al. 2017). A similar conundrum holds for the deeply embedded objects, where the warm water vapor abundance has been traced by $\mathrm{H}_{2}^{18} \mathrm{O}$ observations (e.g., Jacq et al. 1988; van der Tak et al. 2006; Jørgensen \& van Dishoeck 2010; Wang et al. 2012). While there is an indication that the water abundance can be as high as $10^{-4}$ in the warm inner envelope regions $\left(T_{\text {dust }}>100 \mathrm{~K}\right.$, Visser et al. 2013), this is not generally the case. In particular, millimeter interferometric observations of warm water lines $\left(E_{\mathrm{up}} / k_{\mathrm{B}}>200 \mathrm{~K}\right)$ toward a handful of deeply embedded low-mass protostellar systems (Class 0) reveal much lower water abundances than expected on 100 au scales (e.g., Persson et al. 2012). Persson et al. (2016) show that the abundance increases by an order of magnitude after considering that the emission originates from a disk-like structure, part of which is cold $\left(T_{\text {dust }}<100 \mathrm{~K}\right)$, rather than a spherically symmetric envelope. However, the inferred water abundances averaged over 50 au diameter scale are still 1-2 orders of magnitude below the canonical value after taking the physical structure into account. The question of how water is transported from dense clouds to planet-forming disks thus remains open.

In order to understand the water evolution from Class 0 to Class II disks, the fractional water vapor abundance in Class I disks needs to be quantified. By the later Class I stage, Keplerian disks are clearly present and have grown substantially up to $100 \mathrm{au}$ in radius (Harsono et al. 2014; Aso et al. 2015; Yen et al. 2017; Artur de la Villarmois et al. 2019). The large and well-characterized Class I Keplerian disks provide the necessary physical structure to link between the Class 0 and Class II stages of star and planet formation. Class I disks are warmer relative to Class II disks such that the water snowline of Class I disks should be further out and can be spatially resolved with the current millimeter interferometers (Harsono et al. 2015). An additional advantage of Class I objects is the tenuous envelope surrounding the disk that allows for direct observation of water emission from the disk with much less warm inner envelope contribution relative to the Class 0 counterparts.

Jørgensen \& van Dishoeck (2010) and Persson et al. (2012) have shown that the $\mathrm{H}_{2}^{18} \mathrm{O} 3_{1,3}-2_{2,0}$ line $(203 \mathrm{GHz})$ originates from the warm vapor regions of young disks. The Atacama Large Millimeter/submilleter array (ALMA) also opens the window to observe the $\mathrm{H}_{2}^{18} \mathrm{O} 4_{1,4}-3_{2,1}(390 \mathrm{GHz})$ from the ground at high spatial-resolution. Both of these lines have lower Einstein $A$ values than those observed with Herschel (Visser et al. 2013). The lower $A_{\mathrm{ij}}$ value implies that the line is weaker than those targeted by Herschel, but they are less affected by optical depth (both line and dust). Furthermore, by observing the $\mathrm{H}_{2}^{18} \mathrm{O}$ lines, the water emission should be more sensitive to the quiescent gas in the embedded disk than the entrained outflow gas seen in the $\mathrm{H}_{2} \mathrm{O}$ lines (Kristensen et al. 2012; Mottram et al. 2013). Therefore, the $\mathrm{H}_{2}^{18} \mathrm{O}$ lines are suitable to trace the water content in the disk.

This paper presents spatially-resolved water observations toward five Class I protostars with ALMA and NOrthern Extended Millimeter Array (NOEMA). By determining the water abundance in Class I disks, it provides the missing piece in the water evolution from prestellar cores to planet-forming disks. The paper is outlined as follows. Section 2 presents our sources and the details of the observations. The dust continuum emission and water line intensities around the Class I protostars are presented in Sect. 3. Disk masses are determined through the continuum flux densities in Sect. 4. With these masses, we also estimate the average warm water abundance in Class I disks. In order to compare with previous water detections toward Class 0 objects (e.g., Jørgensen \& van Dishoeck 2010; Persson et al. 2012), a similar approach is adopted to obtain upper limits to the water column densities. We discuss the emitting region of warm water lines and their implications in Sect. 5. Finally, the summary and conclusions can be found in Sect. 6 .

\section{Observational details}

\subsection{Class I targets}

We observed five Class I objects in Taurus and Ophiucus molecular clouds (Table 1). Two targets are TMC1A (IRAS 04365+2535) and L1527 IRS (IRAS 04368+2557, hereafter L1527), which are located in the Taurus molecular cloud (Table 1, $d=140$ pc, Elias 1978; Torres et al. 2009). Three additional Class I sources Elias 29 (2MASS J162709432437 187, Elia 2-29), GSS 30 IRS 1 (2MASS J162621382423040 , hereafter GSS30I1), and GSS 30 IRS 3 (2MASS J16262177-2 422 513, hereafter GSS30I3) are embedded protostellar objects in the L1688 core of the $\rho$-Ophiuchi molecular cloud (Table 1, $d=138.4 \pm 2.6$ pc, Mamajek 2008; Ortiz-León et al. 2018). These targets are well-studied embedded protostars with multiwavelength continuum observations that indicate their relatively evolved stage (Chen et al. 1995; Robitaille et al. 2006, see Table 1). Previous molecular gas observations with the Submillimeter Array (SMA) indicated an infalling envelope toward GSS30I1 and an embedded Keplerian disk around Elias 29 (Lommen et al. 2008; Jørgensen et al. 2009). Similarly, an infalling envelope and Keplerian disk has been observed toward TMC1A and L1527 (Ohashi et al. 1997a; Tobin et al. 2012; Harsono et al. 2014; Aso et al. 2015; Yen et al. 2017; van 't Hoff et al. 2018). In terms of ice composition, Elias 29 is particularly interesting since the water gas-to-ice ratio has been determined to be higher than dark clouds $(>0.23$, Boogert et al. 2000). For targets in the Taurus star-forming region, Schmalzl et al. (2014) find high water ice content $\left(N_{\text {ice }} \sim 5 \times 10^{18} \mathrm{~cm}^{-2}\right)$. The regions surrounding these targets are abundant in water ice that can be transported to the disk scales and thermally sublimated in the inner regions of the protostellar systems.

The main difference is that Elias 29, TMC1A, and L1527 have been shown to be surrounded by a Keplerian disk. On the other hand, the physical and chemical structures toward GSS30I1 and GSS30I3 are still unknown. Friesen et al. (2018) find a compact dust disk around both GSS30I1 and GSS30I3 with ALMA (see also Pontoppidan et al. 2002 and Bitner et al. 2008). The ${ }^{12} \mathrm{CO}$ fundamental ro-vibrational lines indicate a molecular emission from a disk wind around GSS30I1 (Herczeg et al. 2011), which indirectly suggests the presence of a Keplerian disk. Meanwhile, the bolometric luminosity of GSS30I3 is much lower than the other targets. The kinematical 
D. Harsono et al.: Missing water in Class I disks

Table 1. Target list and their properties adopted from Bontemps et al. (2001) and Kristensen et al. (2012).

\begin{tabular}{|c|c|c|c|c|c|c|c|c|}
\hline \multirow[b]{2}{*}{ Target } & \multicolumn{2}{|c|}{ Target centers } & \multirow[b]{2}{*}{$\begin{array}{l}L_{\text {bol }} \\
\left(L_{\odot}\right)\end{array}$} & \multirow[b]{2}{*}{$\begin{array}{l}T_{\text {bol }} \\
(\mathrm{K})\end{array}$} & \multirow[b]{2}{*}{$\begin{array}{l}d \\
\mathrm{pc}\end{array}$} & \multirow[b]{2}{*}{$\begin{array}{l}v_{\mathrm{lsr}} \\
\left(\mathrm{km} \mathrm{s}^{-1}\right)\end{array}$} & \multicolumn{2}{|c|}{ Continuum $^{(a)}$} \\
\hline & $\begin{array}{l}\text { RA } \\
\text { (hh:mm:ss) }\end{array}$ & $\begin{array}{l}\text { Dec } \\
\text { (deg:min:sec) }\end{array}$ & & & & & $\begin{array}{c}\text { beam } \\
\theta_{\text {maj }} \times \theta_{\text {min }}(\mathrm{PA})\end{array}$ & $\begin{array}{c}\text { RMS } \\
\left(\mathrm{mJy} \mathrm{beam}^{-1}\right)\end{array}$ \\
\hline \multicolumn{9}{|c|}{ NOEMA: $203 \mathrm{GHz}$} \\
\hline TMC1A & $04: 39: 35.20$ & $25: 41: 44.34$ & 2.7 & 118 & 140 & +6.4 & $0.78 \times 0 . .72\left(62^{\circ}\right)$ & 1.1 \\
\hline L1527 & 04:39:53.88 & 26:03:09.64 & 1.9 & 44 & 140 & +5.9 & $0.78 \times 0.70\left(53^{\circ}\right)$ & 0.86 \\
\hline \multicolumn{9}{|c|}{ ALMA Band 8: $390 \mathrm{GHz}$} \\
\hline Elias 29 & $16: 27: 09.42$ & $-24: 37: 19.19$ & 14.1 & 299 & 138 & +4.3 & $0.39 \times 0.34\left(-75^{\circ}\right)$ & 0.27 \\
\hline GSS30I1 & $16: 26: 21.36$ & $-24: 23: 04.85$ & 13.9 & 142 & 138 & +3.5 & $0.40 \times 0 . .^{\prime} 35\left(-76^{\circ}\right)$ & 4.3 \\
\hline GSS30I3 & $16: 26: 21.70$ & $-24: 22: 50.91$ & 0.13 & $\ldots$ & 138 & $\ldots$ & $0 . .^{\prime} 40 \times 0 . .^{\prime} 35\left(-76^{\circ}\right)$ & 7.9 \\
\hline
\end{tabular}

Notes. Phase centers of the sources and the details on the continuum images are listed. ${ }^{(a)}$ Dust continuum imaging was carried out with natural weighting for the NOEMA data and Briggs weighting (robust =1) for the ALMA data. The noise within each image is calculated in the image plane with an annulus as shown in Appendix A.

evidence of Keplerian disk toward some of the Class I objects (Lommen et al. 2008; Jørgensen et al. 2009; Tobin et al. 2012; Harsono et al. 2014) provides the necessary structure to connect with the Class II disks.

\subsection{NOEMA observations: $p-H_{2}^{18} \mathrm{O} 3_{1,3}-2_{2,0}(203 \mathrm{GHz})$}

TMC1A and L1527 were observed with NOEMA in the B and $\mathrm{C}$ configurations using 6 antennas on 12 January, 9 April, and 19 March 2014 for a total on-source integration time of $6 \mathrm{~h}$. The bandpass calibration was performed on 3C84, 3C454 and J2013+370. Quasars J0507+179 and J04148+380 were used for phase calibration while MWC349 and/or 3C84 were used to bootstrap the amplitude solution. The baseline coverage of these observations is between 11 and $290 \mathrm{k} \lambda$, which translates to a largest scale of $\sim 3000$ au down to 120 au. The spectral setup included one narrow window, $40 \mathrm{MHz}$, targeting the para$\mathrm{H}_{2}^{18} \mathrm{O} 3_{1,3}-2_{2,0}$ transition at $203.4075 \mathrm{GHz}\left(E_{\mathrm{u}} / k=203.68 \mathrm{~K}\right.$, $A_{i j}=4.812 \times 10^{-6} \mathrm{~s}^{-1}$ ) with a spectral resolution of $0.078 \mathrm{MHz}$ $\left(0.12 \mathrm{~km} \mathrm{~s}^{-1}\right)$. A medium resolution window at a spectral resolution of $0.625 \mathrm{MHz}\left(0.92 \mathrm{~km} \mathrm{~s}^{-1}\right)$ was centered at the same location. In addition, two WideX wideband receivers covered $3.6 \mathrm{GHz}$ around the targeted frequency with a spectral resolution of $1.95 \mathrm{MHz}$. Standard calibration and imaging was performed with the GILDAS software ${ }^{1}$. The continuum including the WideX windows was subtracted in the $u v$ space before imaging the water line. The final RMS noise levels in the continuum images are dynamically limited to $1.1 \mathrm{mJy} \mathrm{beam}^{-1}$ for TMC1A and $0.9 \mathrm{mJy} \mathrm{beam}^{-1}$ for L1527 with natural weighting $(0.78 \times 0.72$ beam). Spectral windows (narrow and medium widths) that contain the water lines were imaged with natural weighting to minimize the noise level per velocity channel. Spectra taken with the WideX backend are shown in Appendix A. A spectral cube containing the water line was made at 0.3 and $1 \mathrm{~km} \mathrm{~s}^{-1}$ velocity resolution. Noise levels in $0.3 \mathrm{~km} \mathrm{~s}^{-1}$ channels are 8 and $9 \mathrm{mJy} \mathrm{beam}^{-1}$ for TMC1A and L1527, respectively. The phase centers, beam sizes, and continuum sensitivities are listed in Table 1.

\subsection{ALMA observations: $o-H_{2}^{18} \mathrm{O}_{1,4}-3_{2,1}(390 \mathrm{GHz})$}

Elias 29, GSS330I1, and GSS30I3 were observed with ALMA on 16 June 2015 targeting the ortho- $\mathrm{H}_{2}^{18} \mathrm{O} 4_{1,4}-3_{2,1}\left(E_{\mathrm{u}} / k_{\mathrm{B}}=\right.$ $322.0 \mathrm{~K}, A_{i j}=3.143 \times 10^{-5} \mathrm{~s}^{-1}$ ) line at $390.6078 \mathrm{GHz}$ (project

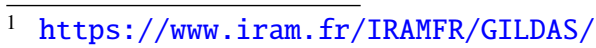

code: 2013.1.00448.S; PI: M. Persson). The observations in Band 8 were carried out with 35 antennas under good weather conditions (precipitation water vapor of $0.5 \mathrm{~mm}$ ). The total on-source integration time was $9.56 \mathrm{~min}$. The final baseline coverage is between 29 and $1020 \mathrm{k} \lambda$ (longest baseline is $783 \mathrm{~m}$ ), which translates to between 40 and $1000 \mathrm{au}$. Unfortunately, the observations toward the GSS30 system used an incorrect phase center such that the objects are located $\sim 12^{\prime \prime}$ away at $\sim 10 \%$ of the primary beam $\left(\sim 15^{\prime \prime} 4\right)$.

A narrow spectral window was dedicated to spectrally resolve the water line with a spectral resolution of $0.061 \mathrm{MHz}$ $\left(0.05 \mathrm{~km} \mathrm{~s}^{-1}\right)$. An additional window was centered on the water line at a lower spectral resolution of $15.625 \mathrm{MHz}\left(\sim 12 \mathrm{~km} \mathrm{~s}^{-1}\right)$. Two other broadband spectral windows were placed around the water transition at a spectral resolution of $15.625 \mathrm{MHz}$ to characterize the continuum emission after removing the bright molecular lines within these windows (see Appendix A). The continuum was subtracted in $u v$ space before imaging the water line.

These nonstandard high frequency observations were manually calibrated with CASA v4.3.1 (McMullin et al. 2007). The spectral windows were combined during the calibration to obtain higher $\mathrm{S} / \mathrm{N}$ on the calibrators. Frequency-averaged gains were solved at $1 \mathrm{~min}$ interval instead of per integration time $(2.02 \mathrm{~s}$, standard calibration) to ensure $S / N>3$. The flux amplitude was calibrated against Titan using $<130 \mathrm{~m}$ baselines (flux $>20 \%$ of maximum). Quasars J1427-4206 and J1625-2527 were used as bandpass and phase calibrators, respectively. In order to characterize the phase at $<1 \mathrm{~min}$ timescales, self-calibration was performed on the continuum for both Elias 29 and GSS 30 (I1 and I3 as point sources). Self-calibration was performed on CASA v5.1.1.

After self-calibration, the dust continuum and the spectral cubes were imaged using the task TCLEAN with Briggs weighting (robust $=1$ ) providing a synthesized beam of $0 .{ }^{\prime} 4 \times 00^{\prime} 35$. Imaging extends to $20 \%$ of the primary field of view for Elias 29 , and down to $0.1 \%$ for GSS30 so as to include both GSS30I1 and GSS30I3. The resulting RMS noise levels in the continuum images are $0.27 \mathrm{mJy}^{\text {beam }^{-1}}$ for Elias 29, $4.3 \mathrm{mJy}^{\text {beam }}{ }^{-1}$ for GSS30I1, and $7.9 \mathrm{mJy}^{\text {beam }^{-1}}$ for GSS30I3 (Table 1). Due to the location of the GSS 30 sources with respect to primary beam, the noise level of their final images is higher than Elias 29. Spectral windows that contain the water lines were imaged with Briggs weighting (robust $=1$ ). Spectral cubes containing the water line were made at 0.3 and $1 \mathrm{~km} \mathrm{~s}^{-1}$ velocity resolutions. 

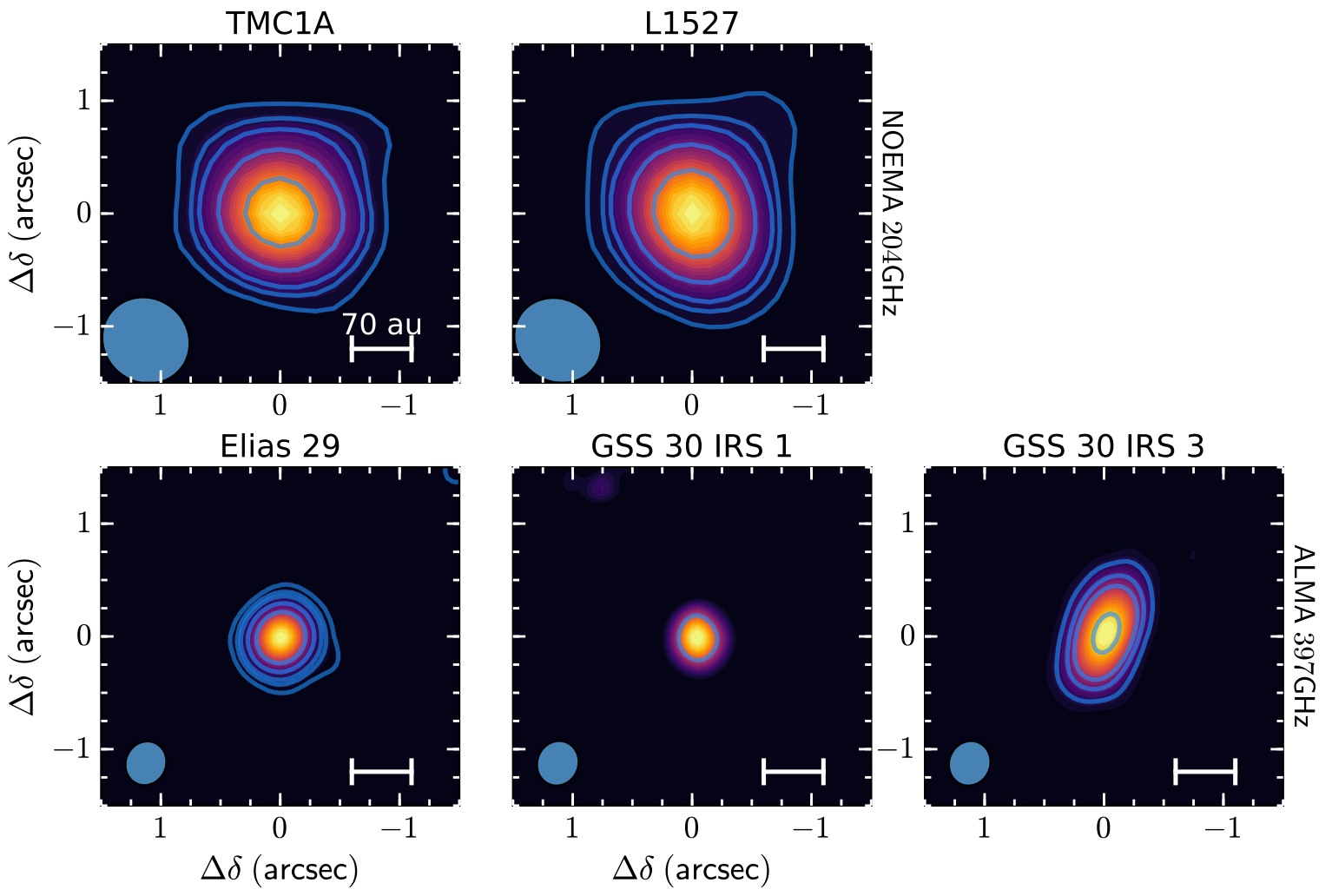

Fig. 1. Dust continuum images centered on the phase-centers listed in Table 1 . The peak intensities are 93, 83, 44, 33, and $278 \mathrm{mJy}^{\mathrm{beam}}{ }^{-1}$ for TMC1A, L1527, Elias 29, GSS30I1, and GSS30I3, respectively. TMC1A and L1527 are imaged at 203.99 GHz (1.4696 mm) while Elias 29, GSS30I1 and GSS30I3 are observed at $397.25 \mathrm{GHz}(754.67 \mu \mathrm{m})$. The color scale spans the dust continuum intensities between $1 \sigma$ to peak intensities with linear spacing. The heavy blue lines indicate the $5,10,15,30$ and $60 \sigma$ contours.

The phase centers, beam sizes and continuum sensitivity of the observations are listed in Table 1.

\section{Dust continuum and water lines observational results}

\subsection{Dust continuum}

Dust continuum emission is detected toward all targets at a $S / N \gtrsim 5$ (Fig. 1). The peak continuum intensities are 93 and $83 \mathrm{mJy} \mathrm{beam}^{-1}$ for TMC1A and L1527, respectively, at $1.5 \mathrm{~mm}$. They are 44, 33, and $278 \mathrm{mJy}^{\text {beam }}{ }^{-1}$ at $750 \mu \mathrm{m}$ for Elias 29 , GSS30I1, and GSS30I3, respectively. Neither disks around TMC1A and L1527 are spatially resolved in our NOEMA images since they do not show the elongation as seen in higher-spatial resolution observations (Harsono et al. 2018; van 't Hoff et al. 2018). Similarly, the continuum around Elias 29 does not show any extended emission as observed in previous molecular gas lines observations (Jørgensen et al. 2009). Our observations are unable to spatially resolve the compact continuum emission toward GSS30I1 ( $\sim 0^{\prime \prime} 4$ beam). GSS30I1 was not detected with the SMA at $1.3 \mathrm{~mm}$ with a beam of $2^{\prime \prime} 8 \times 2$ '. 7 (Jørgensen et al. 2009) while Friesen et al. (2018) and Artur de la Villarmois et al. (2019) detect the unresolved compact component in their $\lesssim 0$ '. $6^{\prime}$ beam. GSS30I3 is spatially resolved showing extended continuum emission in the north-south direction. Previous observations by Jørgensen et al. (2009) detected the molecular gas emission only around GSS30I1, but this is likely associated with the outflow.

To assess the relevant scales that our data are sensitive to, the visibilities amplitude and phase as functions of the projected baselines are shown in Fig. 2. The visibilities for GSS30I1 and GSS30I3 are shown in Appendix A. Phase centers and continuum fluxes are derived by fitting an elliptical Gaussian to the visibilities. The fluxes and phase centers of GSS30I1 and GSS30I3 are derived by fitting 2D Gaussian to their dust continuum image with CASA task imfit in order to take into account the primary beam correction. The results of these fits can be found in Table 1. The continuum flux densities have typical uncertainties of $\sim 20 \%$ for Elias 29, TMC1A and L1527 while the uncertainties are higher $(\sim 40 \%)$ for GSS30I1 and GSS30I3. In comparison to the single-dish $1.1 \mathrm{~mm}$ and $850 \mu \mathrm{m}$ flux densities (Motte \& André 2001; Jørgensen et al. 2009; Kristensen et al. 2012), our observations recover $2-30 \%$ of the total singledish values assuming flux density scaling follows $S_{v} \propto v^{\alpha}$ with $\alpha=2.5$. Our flux densities are consistent with those values reported by Artur de la Villarmois et al. (2019) for Elias 29 and GSS30I1. A decreasing amplitude with increasing $u v$ radius suggests that our observations are sensitive to the physical structure at small-scales $(<1000 \mathrm{au})$ while most of the large-scale emission from the envelope is filtered out.

The derived continuum emission sizes vary between 0.11 to $0 . ' 6$ (Table 2). Meanwhile, the sizes of Keplerian disks around these sources are between 0.'3 and 0.'7 (Lommen et al. 2008; Harsono et al. 2014). The nature of the disk around GSS30I1 is still unknown, however, we take an outer radius of 50 au (0.'35) based on previous fundamental ro-vibrational CO line observations (Pontoppidan et al. 2002; Herczeg et al. 2011). Through the comparison between deconvolved continuum sizes and the extent of the Keplerian disks, our continuum data is dominated by the emission at scales that corresponds to the known Keplerian disks. 


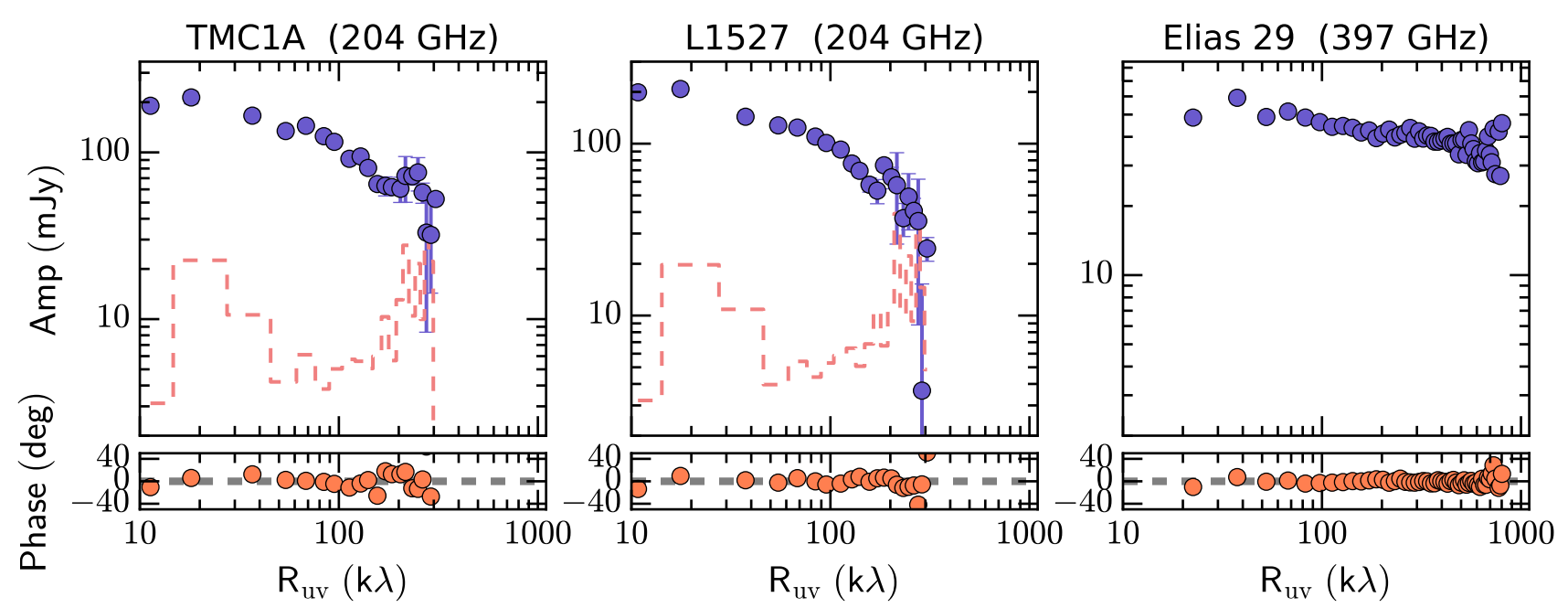

Fig. 2. Circularly averaged binned amplitude (top) and phase (bottom) as functions of projected baselines in $\mathrm{k} \lambda$. Only the visibilities for Elias 29 , TMC1A and L1527 are shown as indicated in the top of each panel while the visibilities toward the GSS30 sources are shown in Appendix A. The standard error of each $u v$ bin, which is smaller than the symbol size, is plotted and the expected zero-signal amplitude is also indicated by the red dashed lines. The expected zero-signal amplitude for Elias 29 is more than a factor of 10 lower than the signal.

Table 2. Dust continuum flux densities and sizes of the sample.

\begin{tabular}{|c|c|c|c|c|c|c|c|}
\hline Target & $\begin{array}{l}F_{0.75 \mathrm{~mm}}{ }^{(a)} \\
(\mathrm{mJy})\end{array}$ & $\begin{array}{l}F_{1.5 \mathrm{~mm}}{ }^{(a)} \\
(\mathrm{mJy})\end{array}$ & $\begin{array}{l}\operatorname{Size}^{(a)} \\
" \times^{\prime \prime}\left({ }^{\circ}\right)\end{array}$ & $\begin{array}{l}F_{1.1 \mathrm{~mm}}{ }^{(b)} \\
(\mathrm{mJy})\end{array}$ & $\begin{array}{l}S_{850 \mu \mathrm{m}}{ }^{(c)} \\
\left(\mathrm{mJy} \mathrm{bm}^{-1}\right)\end{array}$ & $\begin{array}{l}M_{\text {disk }}(d) \\
\left(10^{-3} \times M_{\odot}\right)\end{array}$ & $\begin{array}{l}M_{>100 \mathrm{~K}}{ }^{(d)} \\
\left(10^{-3} \times M_{\odot}\right)\end{array}$ \\
\hline TMC1A & $\ldots$ & $140 \pm 14$ & $0.56 \times 0.44(-59)$ & 256 & 780 & $31(10 \pm 3)$ & 5.5 \\
\hline L1527 & $\ldots$ & $130 \pm 13$ & $0.62 \times 0.44(11)$ & 267 & 1800 & $29(34 \pm 7)$ & 12 \\
\hline Elias 29 & $44 \pm 4$ & $\ldots$ & $0.11 \times 0.08(-2)$ & 109 & 590 & $1.2(2.3 \pm 1)$ & 1.0 \\
\hline GSS30 IRS1 & $37 \pm 10$ & $\ldots$ & $0.44 \times 0.38(89)$ & $\ldots$ & 980 & $1.0(1 \pm 1)$ & 0.7 \\
\hline GSS30 IRS3 & $580 \pm 60$ & $\cdots$ & $0.78 \times 0.43(108)$ & 204 & 980 & $16(14 \pm 4)$ & 2.0 \\
\hline
\end{tabular}

Notes. Disk mass and $100 \mathrm{~K}$ mass around each object are listed. Previous continuum flux density and single-dish measurements are also shown for comparison. ${ }^{(a)}$ Elliptical Gaussian is fit to the visibilities to obtain the continuum flux density, phase center, and deconvolved sizes toward TMC1A, L1527, and Elias 29. The flux densities and continuum sizes of GSS30I1 and GSS30I3 are derived in the image plane by fitting a 2D Gaussian to the intensity profile. We list the 10\% flux error except for GSS30I1 where the RMS noise around the target is higher than the $10 \%$ flux error. ${ }^{(b)}$ Flux density at $1.1 \mathrm{~mm}$ taken from Jørgensen et al. (2009) or extrapolated from $1.36 \mathrm{~mm}$ from Harsono et al. (2014) and Aso et al. (2017) with a flux density frequency dependence of $v^{2.5}$. ${ }^{(c)}$ Peak intensity of the $850 \mu \mathrm{m}$ SCUBA map within a 15" beam from Di Francesco et al. (2008). Since GSS30I1 and GSS30I3 are within 3 pixels in the SCUBA map, the same peak value is listed. The peak SCUBA $850 \mu \mathrm{m}$ intensity toward the phase center of GSS30I1 is $440 \mathrm{mJy}_{\text {beam }}{ }^{-1} .^{(d)}$ Disk mass (gas and dust) derived from ALMA/NOEMA dust continuum fluxes is an average of the masses obtained from varying the dust opacities calculated at $30 \mathrm{~K}$ (see text). The derived disk masses from the power-law disk fit to the visibilities by subtracting the envelope's component (Power-law disk structure) are shown in the parenthesis with their associated $1 \sigma$ errors. The inner warm disk mass, $>100 \mathrm{~K}$, is based on the power-law disk fit to the continuum visibilities with a temperature power-law index $q=0.4$ (Persson et al. 2016, see Appendix B).

\subsection{Water lines}

No water lines are detected toward any of our targets (see Fig. 3 for the $0.3 \mathrm{~km} \mathrm{~s}^{-1}$ spectra). For each target, a spectrum is extracted at the position of the peak continuum intensity and averaged over the dust disk $\left(S_{\text {dust }}>10 \sigma\right)$. By averaging over a larger area, we confirm that the outflow component observed in the ground-state water emission $\left(\mathrm{o}-\mathrm{H}_{2} \mathrm{O}\right.$, Kristensen et al. 2012, Mottram et al. 2014) within the large Herschel beams (39") is not present in these spatially resolved data. Additional stacking analysis on the image plane (e.g., Long et al. 2017) and matched filtering (Loomis et al. 2018) did not extract any water emission from both 0.3 and $1 \mathrm{~km} \mathrm{~s}^{-1}$ spectral cubes. Therefore, we proceed to calculate upper limits to the integrated water line intensities.

There are two useful upper limits that can be quantified from these observations. The first one is the disk-averaged water vapor abundance that can be compared to Class 0 disk-like structures and Class II disks. This value is straightforward to obtain as long as the upper limit to the integrated water flux density is derived from a region within the Keplerian disk. The second quantity is the warm water vapor abundance in the regions inside the water iceline $\left(T_{\text {dust }}>100 \mathrm{~K}\right)$, which we define as the inner warm disk. The abundance in this region is not trivial to obtain directly from observations of embedded protostars (Persson et al. 2016). From the large-scale spherical envelope physical models of Kristensen et al. (2012), the $100 \mathrm{~K}$ region should be inside of 25 au radius. Therefore, we adopt 25 au as the radius over which to derive an upper limit of water in the inner warm disk component. By adopting a 25 au radius, the water vapor column density in Class I protostellar systems can be compared to Class 0 observations ( $\sim 25$ au radius emitting region; Jørgensen $\&$ van Dishoeck 2010; Persson et al. 2012).

Two spatial masks ( $x, y$ pixels) are used to calculate the upper limits to the integrated line flux density $\left(\mathrm{Jy} \mathrm{km} \mathrm{s}{ }^{-1}\right.$ ): one over the dust continuum size ( $>5 \sigma$, disk average) and a circular mask of 25 au radius ( $\sim$ !' 2 , inner warm disk). We note that the 

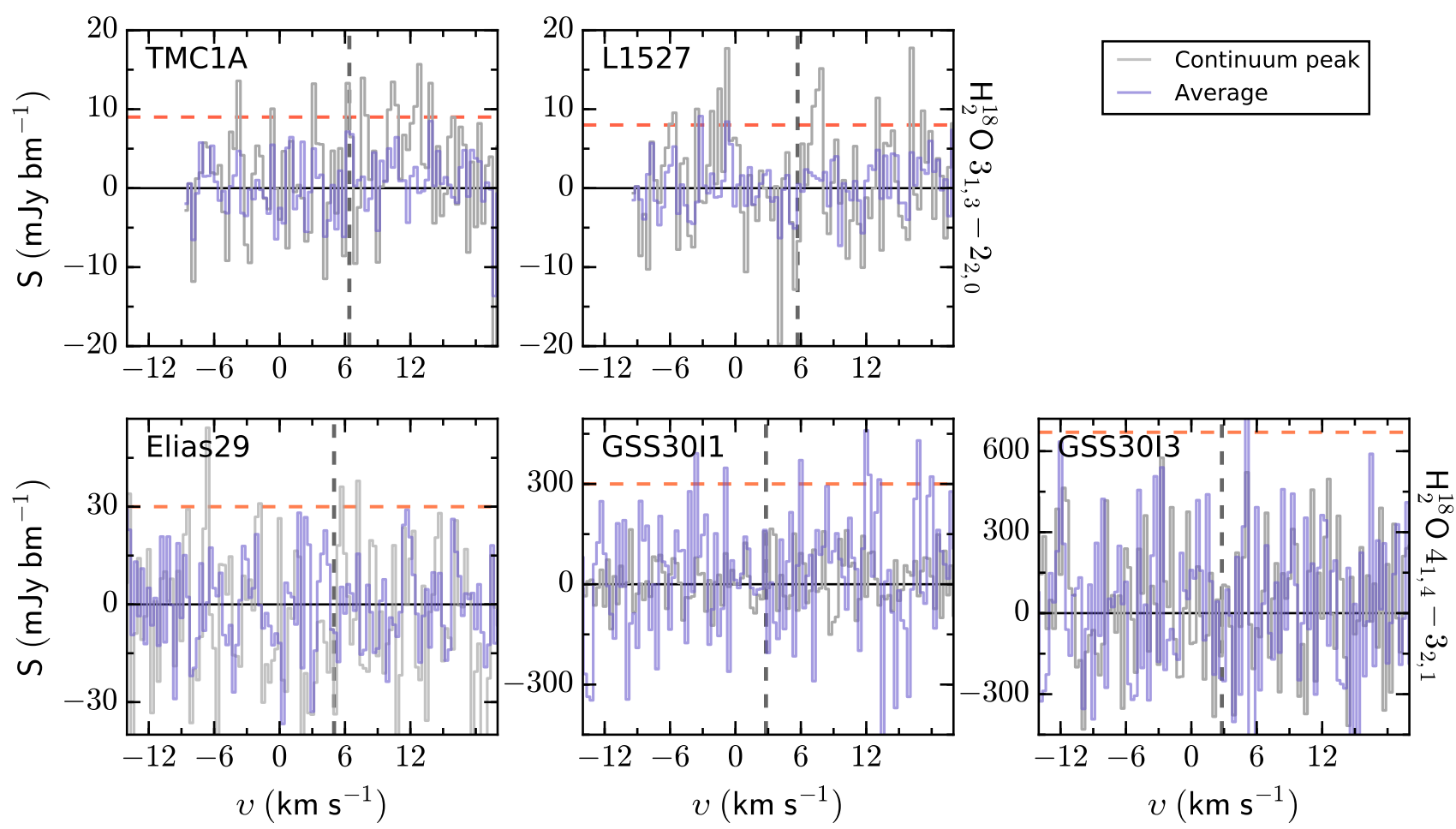

Fig. 3. Water spectra toward the Class I young stellar objects. Spectra extracted toward the continuum peak are shown by the gray lines, and the average spectra extracted from a region defined by $>10 \sigma$ in the dust continuum image is indicated in blue. Vertical black dashed line shows the systemic velocity of the source. Horizontal red line shows the $1 \sigma$ noise level while the black solid line indicates the baseline.

Table 3. Synthesized beams and RMS noise level per $0.3 \mathrm{~km} \mathrm{~s}^{-1}$ channel for the water line images.

\begin{tabular}{|c|c|c|c|c|c|c|c|c|}
\hline \multirow[b]{2}{*}{ Target } & \multirow[b]{2}{*}{ Beam } & \multirow[b]{2}{*}{$\begin{array}{l}\text { Noise/channel } \\
\left(\mathrm{mJy}_{\text {beam }}^{-1}\right)\end{array}$} & \multicolumn{3}{|c|}{ Disk average quantities } & \multicolumn{3}{|c|}{ Inner warm disk } \\
\hline & & & $\begin{array}{l}\int S_{v} \mathrm{~d} v \\
(\mathrm{mJy}) \\
\left(\mathrm{km} \mathrm{s}^{-1}\right)\end{array}$ & $\begin{array}{l}N_{\mathrm{H}_{2}^{18} \mathrm{O}} \\
\left(\mathrm{cm}^{-2}\right)\end{array}$ & $N_{\mathrm{H}_{2} \mathrm{O}} / N_{\mathrm{H}_{2}}$ & $\begin{array}{l}\int S_{v} \mathrm{~d} v \\
(\mathrm{mJy}) \\
\left(\mathrm{km} \mathrm{s}^{-1}\right)\end{array}$ & $\begin{array}{l}N_{\mathrm{H}_{2}^{18} \mathrm{O}} \\
\left(\mathrm{cm}^{-2}\right)\end{array}$ & $N_{\mathrm{H}_{2} \mathrm{O}} / N_{\mathrm{H}_{2}}$ \\
\hline TMC1A & $0.79^{\prime \prime} \times 0.72^{\prime \prime}\left(59^{\circ}\right)$ & 9.0 & $<11$ & $<4 \times 10^{14}$ & $<1 \times 10^{-7}$ & $<3$ & $<1 \times 10^{14}$ & $<7 \times 10^{-7}$ \\
\hline L1527 & $0.79^{\prime \prime} \times 0.71^{\prime \prime}\left(53^{\circ}\right)$ & 8.0 & $<10$ & $<3 \times 10^{14}$ & $<2 \times 10^{-7}$ & $<3$ & $<1 \times 10^{14}$ & $<8 \times 10^{-7}$ \\
\hline Elias 29 & $0.39^{\prime \prime} \times 0.31^{\prime \prime}\left(-76^{\circ}\right)$ & 30 & $<25$ & $<3 \times 10^{14}$ & $<8 \times 10^{-7}$ & $<17$ & $<2 \times 10^{14}$ & $<1 \times 10^{-5}$ \\
\hline GSS30 IRS1 & $0.41^{\prime \prime} \times 0.32^{\prime \prime}\left(-75^{\circ}\right)$ & 300 & $<200$ & $<2 \times 10^{15}$ & $<1 \times 10^{-6}$ & $<200$ & $<2 \times 10^{15}$ & $<1 \times 10^{-4}$ \\
\hline GSS30 IRS3 & $0.41^{\prime \prime} \times 0.33^{\prime \prime}\left(-75^{\circ}\right)$ & 670 & $<890$ & $<9 \times 10^{15}$ & $<2 \times 10^{-6}$ & $<52$ & $<5 \times 10^{15}$ & $<3 \times 10^{-5}$ \\
\hline
\end{tabular}

Notes. The upper limit to the integrated water line intensities are listed for a Gaussian line with a $F W H M$ of $1 \mathrm{~km} \mathrm{~s}^{-1}$ (see text). Upper limits are extracted for the region of the disk and the inner warm disk region $\left(T_{\text {dust }}>100 \mathrm{~K}\right)$ as indicated below.

deconvolved Band 8 dust continuum size of Elias 29 is less than $25 \mathrm{au}$, however the continuum sizes at longer wavelengths are larger. The emitting size at $0.87 \mathrm{~mm}$ is $0.17^{\prime \prime} \times 0.16^{\prime \prime}(\sim 24 \mathrm{au}$ diameter, Artur de la Villarmois et al. 2019) while it is $\sim 2^{\prime \prime}$ at $1.1 \mathrm{~mm}$ ( $\sim 140$ au diameter, Lommen et al. 2008; Jørgensen et al. 2009). Therefore, the cold dusty disk is more extended than our Band 8 observations. A spectrum is extracted over the pixels within each of the spatial mask following Carney et al. (2019),

$\sigma_{v}(J y)=\sqrt{\frac{\sum(x, y)}{n_{\mathrm{ppb}}}} \sigma_{\mathrm{rms}}\left(\mathrm{Jy}_{\mathrm{beam}}{ }^{-1}\right)$,

where $n_{\mathrm{ppb}}$ is the number of pixels per beam to correct for the correlated noise within the beam and $\sigma_{\text {rms }}$ is the RMS noise per channel in mJy beam ${ }^{-1}$ (Table 3 ). Since the underlying velocity pattern of the water lines is not known toward these systems due to presence of disk winds (e.g., Herczeg et al. 2011; Bjerkeli et al. 2016), we assume that the underlying line profile is Gaussian. The number of channels $N_{\text {chan }}$ that are being considered in the calculation corresponds to a Gaussian linewidth (FWHM) of $1 \mathrm{~km} \mathrm{~s}^{-1}$ based on the width of the $\mathrm{H}_{2}^{18} \mathrm{O}$ line observed toward the Class 0 objects ( $\sim 3$ channels, Persson et al. 2014). An upper limit is set at $3 \sigma$ where $\sigma=\sigma_{v} \sqrt{N_{\text {chan }}} \delta v$ in Jy $\mathrm{km} \mathrm{s}^{-1}$ with $\delta v$ as the velocity width. These upper limits to the integrated water flux densities for both disk average and the inner warm disk values can be found in Table 3 .

\section{Upper limits to the water vapor abundance}

Upper limits to the average warm water abundance are estimated by normalizing the warm water column density by the $\mathrm{H}_{2}$ column density. The water column density upper limit $N_{\mathrm{H}_{2}^{18} \mathrm{O}}$ is derived using the upper limits obtained in the previous section. 
First, we present the disk masses calculated from the dust continuum flux densities and through the analysis of the continuum visibilities after the removal of the large-scale envelope component. Then, we calculate the $\mathrm{H}_{2}$ column density from the disk mass in order to derive the water abundance.

\subsection{Total disk mass: gas + dust}

Disk masses (gas + dust) are calculated from the dust continuum fluxes prior to the removal of the large-scale envelope contribution (Table 1) by adopting an average dust temperature and a dust mass absorption coefficient. We explore a range of dust opacities $\left(\kappa_{v}\right.$ between $0.7-2.4 \mathrm{~cm}^{2} \mathrm{~g}^{-1}$ at $204 \mathrm{GHz}$ and $2.2-5.0 \mathrm{~cm}^{2} \mathrm{~g}^{-1}$ at $397 \mathrm{GHz}$, Beckwith et al. 1990; Ossenkopf \& Henning 1994; Andrews et al. 2009; Bruderer et al. 2012) to reflect the large grain sizes implied by cm-wavelengths observations of L1527 (Melis et al. 2011) and Elias 29 (Miotello et al. 2014). An average $\kappa_{v}$ is used to derive the total disk mass using the formula (Hildebrand 1983; Beckwith et al. 1990)

$M_{\text {dust }}=\frac{S_{v} d^{2}}{\kappa_{v} B_{v}\left(T_{\text {dust }}\right)}$,

with a dust temperature $T_{\text {dust }}$ of $30 \mathrm{~K}$ and gas-to-dust ratio of 100 to obtain the total disk mass (gas and dust) that is tabulated in Table 2.

While our disk masses derived at $1.1 \mathrm{~mm}$ (TMC1A and L1527) are similar to previous results (e.g., Jørgensen et al. 2009), we obtain lower masses for observations at $750 \mu \mathrm{m}$ (Elias 29, GSS30I1, and GSS30I3) by more than a factor of 2 . For Elias 29, Jørgensen et al. (2009) find a disk mass of $0.011 M_{\odot}$ while we obtain a disk mass of $\sim 0.001 M_{\odot}$ (a factor of 10 difference). In comparison with Friesen et al. (2018), the disk mass of GSS30I3 is within a factor of two while it is within a factor of four for GSS30I1. Artur de la Villarmois et al. (2019) adopt a temperature of $15 \mathrm{~K}$ (Dunham et al. 2014) to calculate the mass of the disk around Elias 29 and GSS30I1 to get 6 times higher values. It is likely that the disk mass derived from the flux density at $750 \mu \mathrm{m}$ is a lower limit due to optically thick compact dust emission.

Power-law disk structure. A disk mass derived from a single temperature (Eq. (1)) is not sufficient to characterize the water emitting mass $\left(T_{\text {dust }}>100 \mathrm{~K}\right)$. In order to estimate the small-scale structure ( $<100$ au, Lay et al. 1997), the dust continuum visibilities are fit using the methodologies presented in Persson et al. (2016, see also Appendix C) to provide independent measures on the disk mass and the water emitting mass. A power-law spherical envelope model (Kristensen et al. 2012, Appendix B) has been used to predict the large-scale $\left(>5^{\prime \prime}\right.$, $<50 \mathrm{k}$ ) contribution to the continuum emission. A power-law disk structure as described by a surface density profile $\left(\Sigma \propto R^{-1}\right)$ and a dust temperature profile $\left(T_{\text {dust }} \propto R^{-q}\right)$ is fitted to the visibilities after subtracting the large-scale envelope component. Using this procedure, we obtain similar (within a factor of 2) disk masses as listed in Table 2.

With these methodologies, the $100 \mathrm{~K}$ mass is estimated for each object and tabulated in Table 2 for a power-law index $q=0.4$, which is expected for an irradiated embedded disk (e.g., van 't Hoff et al. 2018). By changing the temperature power-law index $q$, the $100 \mathrm{~K}$ mass varies within a factor of 3 . A flatter $q(0.35)$ leads to a significant fraction of the disk to be above $100 \mathrm{~K}$, while the $100 \mathrm{~K}$ boundary shifts inward to smaller radii for a steeper $q(0.5)$. The total mass of the disk is lower if the entire disk is warm (e.g., $q=0.3$ ) since less material is needed to reproduce the observed intensity profile.
Using the derived masses, we can calculate both the $\mathrm{H}_{2}$ column densities $N_{\mathrm{H}_{2}}$ for the entire disk and in the inner warm disk using

$N_{\mathrm{H}_{2}}=\frac{M_{\mathrm{disk}}}{d A \mu_{\mathrm{H}_{2}} m_{\mathrm{p}}}$,

where $\mu_{\mathrm{H}_{2}}=2.8$ (Kauffmann et al. 2008) and averaged over an area $d A$. An appropriate mass for a region encompassing the dust disk (within $>10 \sigma$ contours) and the inner warm disk ( $25 \mathrm{au}$ ) by changing the area $d A$ and correcting for the mass fraction. For these calculations, we adopt a disk whose temperature profile is proportional to $R^{-0.4}$. By applying these methods, we also get a better handle on disk masses after considering the large-scale envelope's contribution.

\subsection{Upper limits to disk averaged water vapor abundance}

In order to compare our observations to the spatially resolved water observations toward Class 0 protostellar systems, we adopt the same method to derive the water column density. An estimate is obtained by considering thermalized and optically thin water emission through (Goldsmith \& Langer 1999)

$N_{\mathrm{H}_{2}^{18} \mathrm{O}}\left(\mathrm{cm}^{-2}\right)=\frac{8 \pi k_{\mathrm{B}} v^{2}}{A_{i j} h c^{3}} \frac{Q_{\mathrm{rot}}\left(T_{\mathrm{ex}}\right)}{g_{\mathrm{u}}} \exp \left(\frac{E_{\mathrm{u}}}{T_{\mathrm{ex}}}\right) \mathcal{G} \int S_{v} \mathrm{~d} v$,

where the partition function $Q_{\text {rot }}$ is obtained from the Cologne Database for Molecular Spectroscopy (Müller et al. 2005; Endres et al. 2016) that accounts for the temperature dependent orthoto-para ratio, an excitation temperature $T_{\mathrm{ex}}$ of $200 \mathrm{~K}$ (Coutens et al. 2014), the gain factor $\mathcal{G}=\frac{\lambda^{2}}{2 k_{\mathrm{B}} \Omega}\left(\mathrm{K} \mathrm{Jy}^{-1}\right)$ at the observed wavelength $\lambda$, Boltzmann constant $k_{\mathrm{B}}$, beam solid angle $\Omega$, and the integrated line flux density $\int S_{v} \mathrm{~d} v$. Inserting the upper limits into the equation above, we derive upper limits on the $\mathrm{H}_{2}^{18} \mathrm{O}$ column densities of $\sim 3 \times 10^{14} \mathrm{~cm}^{-2}$ for both TMC1A and L1527 averaged over the entire dust disk (see Table 3). Similarly, the $3 \sigma$ upper limit to the $\mathrm{H}_{2}^{18} \mathrm{O}$ column densities for Elias 29, GSS30I1, and GSS30I3 are $2.9 \times 10^{14}, 2.1 \times 10^{15}$, and $9.4 \times 10^{15} \mathrm{~cm}^{-2}$, respectively. Table 3 lists these upper limits to the $\mathrm{H}_{2} \mathrm{O}$ column densities adopting a ${ }^{16} \mathrm{O} /{ }^{18} \mathrm{O}=540$ (Wilson \& Rood 1994).

Upper limits to the disk-averaged water vapor abundance are calculated by dividing the $\mathrm{H}_{2} \mathrm{O}$ column density by the total $N_{\mathrm{H}_{2}}$ using the entire disk mass. These values are between $1 \times 10^{-7}$ and $10^{-6}$ (see Table 3 ). These water abundances are much lower than the canonical value of $10^{-4}$ with respect to $\mathrm{H}_{2}$ averaged over the entire disk.

\subsection{Upper limits to the averaged water vapor abundance in the inner warm disk}

Most of the water vapor is inside of the water iceline at $\sim 100 \mathrm{~K}$ (inner warm disk). While other regions in an embedded system may have some water vapor, our $\mathrm{H}_{2}^{18} \mathrm{O}$ observations are particularly sensitive to the inner warm disk component (see Sects. 4.4 and 5.2). This section mainly focuses on the warm disk component. As a zeroth-order approximation, the water iceline is proportional to the bolometric luminosity. For most of these systems, their dust temperature structure reaches $100 \mathrm{~K}$ at $\sim 25$ au from the protostar while it is $\lesssim 3$ au for GSS30I3 due to its lower bolometric luminosity (e.g., Harsono et al. 2015). Since these scales are located well within the Keplerian disk, the $100 \mathrm{~K}$ mass can be scaled from the total disk mass by considering a powerlaw disk whose surface mass density follows $\Sigma \propto R^{-1}$ and an outer radius of $100 \mathrm{au}$ for simplicity. The mass within 25 au 
Table 4. Properties of low-mass protostellar systems and their warm water column densities.

\begin{tabular}{llllll}
\hline \hline Target & $\begin{array}{l}L_{\text {bol }} \\
\left(L_{\odot}\right)\end{array}$ & $\begin{array}{l}M_{\text {env }} \\
\left(M_{\odot}\right)\end{array}$ & $\begin{array}{l}M_{\text {disk }} \\
\left(M_{\odot}\right)\end{array}$ & $\begin{array}{l}\text { Size } \\
\left({ }^{\prime \prime}\right)\end{array}$ & $\begin{array}{l}N_{\mathrm{H}_{2} \mathrm{O}}{ }^{(a)} \\
\left(\mathrm{cm}^{-2}\right)\end{array}$ \\
\hline Class 0 \\
IRAS 2A & 35.7 & 5.1 & 0.06 & 1 & $6.3 \times 10^{19}$ \\
IRAS 4A NW & 9.1 & 5.6 & 0.05 & 1 & $1.7 \times 10^{19}$ \\
IRAS 4B & 4.4 & 3.0 & 0.14 & 0.8 & $8.4 \times 10^{18}$ \\
IRAS 4A SE & 9.1 & 5.6 & 0.09 & 1 & $<5.8 \times 10^{17}$ \\
TMC1A & 2.7 & 0.2 & 0.031 & 0.5 & $<7.3 \times 10^{17}$ \\
L1527 & 1.9 & 0.9 & 0.029 & 0.5 & $<6.9 \times 10^{17}$ \\
Elias 29 & 14.1 & 0.04 & 0.001 & 0.1 & $<2.1 \times 10^{17}$ \\
GSS30I1 & 13.9 & 0.1 & 0.001 & $<0.4$ & $<1.5 \times 10^{18}$ \\
GSS30I3 & 0.13 & 0.1 & 0.016 & 0.78 & $<6.8 \times 10^{18}$ \\
\hline
\end{tabular}

Notes. Values are taken from Kristensen et al. (2012) and Persson et al. (2014). ${ }^{(a)}$ Water column densities within 25 au radius.

is $\sim 25 \%$ of the total disk mass while it is $3 \%$ for $3 \mathrm{au}$. The derived upper limits to the water vapor abundance are between $7 \times 10^{-7}$ and $1 \times 10^{-5}$ averaged over the inner warm disk ( $25 \mathrm{au,}$ see Table 3). The upper limits for the GSS30 sources are higher, however, the data toward this region are less sensitive than the other regions. By scaling the disk mass according to this simple method, the average water abundance increases by a factors of 4 up to an order of magnitude excluding the GSS30 objects.

A more sophisticated method is to use the $100 \mathrm{~K}$ mass obtained from the parametric disk model (power-law disk structure). The difference on the average warm water vapor abundance compared with the simple method is only significant for GSS30I1.

\subsection{Optical depth effects and other possible caveats}

While water emission is not detected toward our targets, spatially resolved warm $\mathrm{H}_{2}^{18} \mathrm{O}$ emission has been detected toward Class 0 protostars. The main difference between Class 0 and Class I protostellar objects is the envelope mass (see Table 4). Thus far, the water emission is detected toward Class 0 objects that are surrounded by a $>1 M_{\odot}$ envelope. Since the emitting mass is the dominant component, the optical depth of both the dust and line may influence the strength of water emission. The water line opacity is higher for Class 0 protostars than their Class I counterparts simply due to the higher water column density (Table 4). In order to examine the dust continuum optical depth effect, we take the disk mass divided by the dust continuum size using the values in Table 4. On average, this approximation suggests that the dust optical depth at both 203 and $390 \mathrm{GHz}$ is a factor of 2 higher for the Class I disks relative to Class 0 disks mostly due to their observed smaller size. Thus, the millimeter water line emission for Class I protostars could be attenuated by dust.

In order to place our observations in the context of star and disk formation, the general water vapor reservoirs need to be defined. Those within Class II disks have been studied in detail (e.g., Woitke et al. 2009; van Dishoeck et al. 2014; Notsu et al. 2016). Water vapor is located in three regions. In region 1 , the water vapor is in the midplane $(z / R<1)$ and in the inner regions of disks up to the dust sublimation radius $\left(160<T_{\text {dust }}<1500 \mathrm{~K}\right)$ where the density is high. The water vapor in region 2 originates from the nonthermal desorption of water since the dust temperature is low in the outer disk $(R>20$ au, $\left.T_{\text {dust }}<100 \mathrm{~K}\right)$. Meanwhile, the water vapor in region 3 is located in the warm upper layer of disks $(R<20 \mathrm{au}, z / R>0.1)$ where $T_{\text {gas }}>T_{\text {dust }}$. In terms of water abundance, region 1 has the highest water vapor abundance at $10^{-4}$ with respect to $\mathrm{H}_{2}$ while it is $\lesssim 10^{-5}$ in region 3 . Since most of the water is frozen out at $R>20$ au (region 2), the predicted fractional water vapor abundance as a result of photodesorption is low there.

Despite the distinct water vapor reservoirs, it is not straightforward to relate the observed water lines to the specified regions. While region 1 has the most water vapor, it is difficult to observe directly because it is located inside the optically thick region in the continuum. The water vapor in region 3 has been observed through hot $\mathrm{H}_{2}^{16} \mathrm{O}$ lines in the infrared (e.g., Zhang et al. 2013; Fedele et al. 2013; Antonellini et al. 2015). The cold water reservoir that resides in region 2 can only be observed through the ground state water lines $\left(\mathrm{H}_{2}^{16} \mathrm{O}\right)$ at 556 and $1113 \mathrm{GHz}$, which indeed indicate very low water vapor abundances (e.g., Hogerheijde et al. 2011; Du et al. 2017).

It is instructive to connect the water reservoirs in embedded systems to the Class II disks. An embedded protostellar system is comprised of a molecular outflow, protostellar envelope, and a disk. For Class 0 objects, the disk is typically called a disk-like structure since the kinematical structure as inferred from $\mathrm{C}^{18} \mathrm{O}$ observations is non-Keplerian. Water emission has been observed from the outflow component in young protostars (e.g, Kristensen et al. 2012; Tafalla et al. 2013). It is characterized by broad emission lines $\left(F W H M>10 \mathrm{~km} \mathrm{~s}^{-1}\right)$. The narrow $\mathrm{H}_{2}^{18} \mathrm{O}$ lines $\left(<5 \mathrm{~km} \mathrm{~s}^{-1}\right)$ that are detected toward young embedded systems with both Herschel (Visser et al. 2013) and NOEMA (Persson et al. 2012) indicate the presence of quiescent gas corresponding to the protostellar envelope and embedded disk. Using radiative transfer models of embedded disks, Harsono et al. (2015) suggest that most of the observed $\mathrm{H}_{2}^{18} \mathrm{O}$ emission toward Class 0 objects is due to the surrounding warm inner envelope including the disk-like structure. A selfconsistent physical and chemical disk+envelope model is needed to disentangle the two contributions and determine the exact water abundance structure in the inner disk regions of embedded objects.

This paper presents the nondetection of $\mathrm{H}_{2}^{18} \mathrm{O}$ lines in Class I disks. Since the envelope mass of our targets is low $\left(<1 M_{\odot}\right)$, the contribution from the surrounding envelope should also be much lower than for Class 0 protostellar systems. Figure 4 shows the predicted $\mathrm{H}_{2}^{18} \mathrm{O}$ line from an embedded system (a $0.02 M_{\odot}$ disk surrounded by a $1 M_{\odot}$ envelope irradiated by a central $1 L_{\odot}$ star). Our upper limits are consistent with the expected water emission from the embedded disk only (no envelope) with water emission from a water vapor rich envelope ruled out. The figure also shows that the upper limits are consistent with a small percentage of the disk that can contribute to the water emission. Since the water column densities in region 3 are low, it is unlikely that $\mathrm{p}-\mathrm{H}_{2}^{18} \mathrm{O}$ emission can be detected from the region, given also the ${ }^{16} \mathrm{O} /{ }^{18} \mathrm{O}$ isotope ratio of 540 and ortho-to-para ratio of 3. Moreover, the surface layers in region 3 have lower gas densities, making it less effective in emitting photons. Although the critical density of the line is moderate, the observed line flux limits the emitting region to $\sim 0$.' 1 . In addition, line opacity and pumping dust continuum increase the molecular excitation, further reducing the size of the emitting region to satisfy the given line flux. Following the standard picture of water reservoirs as outlined above, the $\mathrm{H}_{2}^{18} \mathrm{O}$ observations are therefore sensitive only to the warm water vapor reservoir inside of region 1 (inner warm disk, $T>100 \mathrm{~K}$ ) 


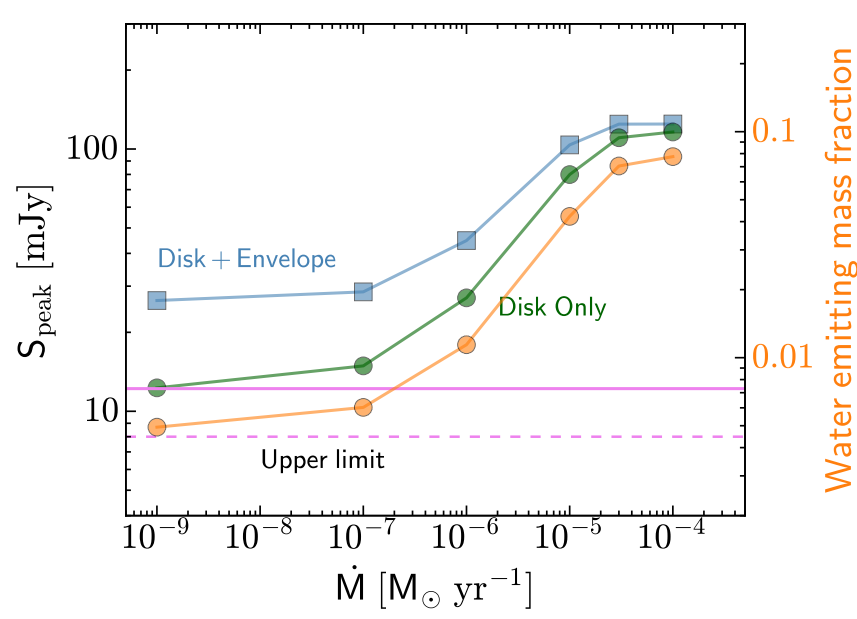

Fig. 4. Peak flux density of the $\mathrm{H}_{2}^{18} \mathrm{O} 3_{1,3}-2_{2,0}(203 \mathrm{GHz})$ as function of the stellar accretion rate. These models are based on the embedded disk models of Harsono et al. (2015) with a central luminosity of $1 L_{\odot}$. The blue squares indicate the predicted water emission by taking into account water vapor inside the disk and envelope. The green circles show the expected water flux densities emitted only by the embedded disk. These models adopt a water vapor abundance of $10^{-4}$ in the regions where $T_{\text {dust }}>100 \mathrm{~K}$ and visual extinction $A_{\mathrm{v}}>3$ to avoid regions whose emission can be affected by outflowing gas. The predicted line flux densities are calculated via thermalized molecular emission (Eqs. (9) and (10) of Harsono et al. 2015) considering the water column density inside of 25 au radius. From these embedded disk models, the water emitting mass fraction is shown as function of the accretion rate in orange. The upper limit $(1 \sigma)$ for our observations is indicated by the horizontal purple dashed line. The full radiative transfer of water from a generic disk model (see text in Sect. 5.4, Bosman et al., in prep.) is indicated by the purple line to indicate the integrated water flux density for a typical disk in the absence of and accretion heating.

that has an expected abundance of $10^{-4}$ inside the optically thick region.

Our adopted analysis is heavily dependent on the simplified radiative transfer of water. Recently, Notsu et al. (2016) present calculations of the strength of water lines from protoplanetary disks that include water chemistry and thermalized water emission. We have used a generic protoplanetary disk model (Bosman et al., in prep.) that has a similar complexity as Notsu et al. (2016) including nonlocal thermal equilibrium calculation and dust continuum radiative transfer. The generic disk model is akin to the model of the AS $205 \mathrm{~N}$ disk $\left(0.03 M_{\odot}\right.$ disk, $L=7 L_{\odot}$, Bruderer et al. 2015), which is roughly the mass of the embedded disks in our sample. The predicted strength of the $\mathrm{H}_{2}^{18} \mathrm{O}$ $3_{1,3}-2_{2,0}(203.4 \mathrm{GHz})$ is $12.2 \mathrm{mJy}$ as indicated by the purple line in Fig. 4. It provides limits on the strength of the water line in the absence of surrounding envelope and accretion heating with a higher central luminosity $\left(1 L_{\odot}\right.$ vs. $\left.7 L_{\odot}\right)$.

\section{Origin of the low warm water vapor abundance and its implication}

One of the missing pieces of the water trail from pre-stellar cores to planet-forming disks is the water abundance in Class I disks. It is known that water ice is abundant in pre-stellar cores (Boogert et al. 2015). Meanwhile, some low-mass Class 0 sources already show surprisingly low warm water vapor abundances (Persson et al. 2012). In order to trace the water evolution during star and planet formation, Class I sources are prime targets since they have warm Keplerian disks surrounded by a tenuous envelope.
Since the data toward GSS30 are much less sensitive than the other data sets, we have excluded these from further discussions. The remaining data provide the most stringent upper limit of the water abundance toward newly formed planet-forming disks at least in regions that are warm enough to have water vapor. With these data, the water abundance averaged over Class I Keplerian disks is much lower than expected if the water abundance were $10^{-4}$ over the inner 25 au radius, by at least a factor of 10 .

\subsection{Water vapor emitting regions}

To constrain the amount of water and its location, it is instructive to create a simple picture of the water emitting regions. From previous results, most of the quiescent water vapor in Class 0 protostars is located in the inner warm envelope (e.g., Jørgensen \& van Dishoeck 2010; Mottram et al. 2013; Harsono et al. 2015). Meanwhile, if we consider the older Class II disks, physical and chemical models have been used to indicate the water reservoirs (e.g., Glassgold et al. 2009; Bethell \& Bergin 2009; Woitke et al. 2010; Bergin \& van Dishoeck 2012; Walsh et al. 2015; Du et al. 2017, Sect. 4.4). Most of the water in Class II disks resides near the midplane in the inner few au where it is invisible (e.g., Carr \& Najita 2008; Meijerink et al. 2009) while the water vapor is frozen-out and located in the photodesorbed layer at the outer disk. Our results suggest that the water vapor in Class I sources probed by $\mathrm{H}_{2}^{18} \mathrm{O}$ mm-data originates from regions that are more common to the Class II disks than Class 0 protostars. Based on these studies, it is now possible to highlight the water vapor emitting regions for the different stages of low-mass star formation as shown in Fig. 5.

The high water abundance region in Class I disks most likely resides in the Keplerian disk rather than envelope. However, it is not entirely clear if the water line emission can trace Keplerian motion. Disks embedded in an infalling envelope, in general, are still more active than Class II disks (e.g., Vorobyov \& Basu 2005; Harsono et al. 2011; Kratter \& Lodato 2016). Recent ALMA observations show evidence of such activities: infalldriven instabilities (e.g., Pérez et al. 2016; Hall et al. 2018; Lee et al. 2020) and disk winds (e.g., Herczeg et al. 2011; Bjerkeli et al. 2016; Tabone et al. 2017). Without a detection of spectrally resolved water lines, it is difficult to conclude that the molecular emission would be strictly Keplerian. For this reason, we have simply assumed in our analysis in Sects. 3 and 4 that the water line is Gaussian similar to the observed line profile toward Class 0 objects.

\subsection{Water abundance across evolutionary stage}

Our observations are sensitive to the compact disks at $100 \mathrm{au}$ scales. We also showed that these data are sensitive to physical scales well within the known Keplerian disks $(R<100 \mathrm{au})$. Therefore, we find that the average water abundance in young protoplanetary disks is much lower than the canonical value of $10^{-4}$ with respect to $\mathrm{H}_{2}$. Such a high value is expected if a significant fraction of the young disk inside of 25 au radius is warm enough such that water ice sublimates.

Water emission has been detected toward the luminous Class 0 protostellar objects with bolometric luminosities between 4 to $25 L_{\odot}$. The Class I objects in our sample are only slightly less luminous with bolometric luminosities between 1.9 and $14 L_{\odot}$. Thus, luminosity alone cannot explain the nondetections of water lines toward the targeted Class I objects. For example, Elias 29 is more luminous than IRAS 4A and IRAS 4B while water emission is detected toward both IRAS 4 sources in the NGC 1333 region but not toward Elias 29. Therefore, our nondetections 

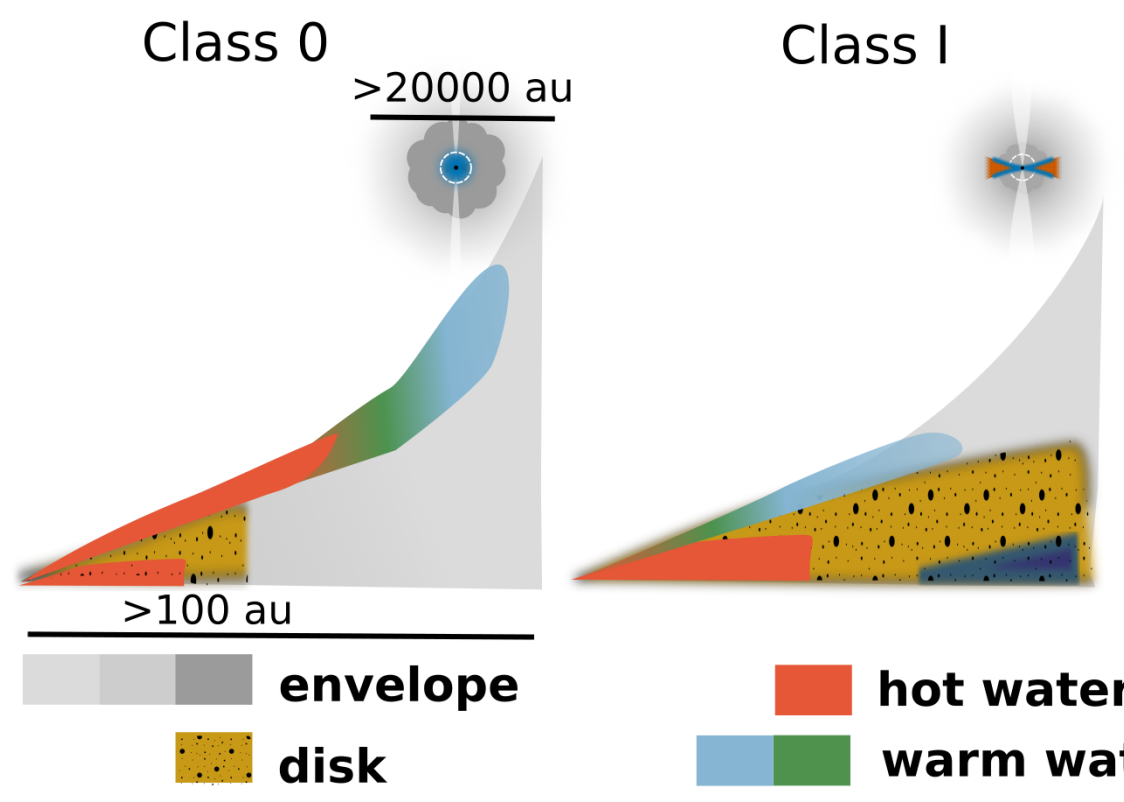

Class II

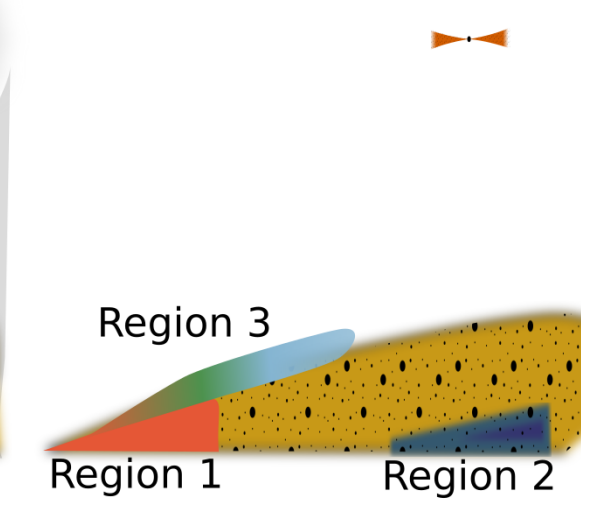

Fig. 5. Schematic drawing of the water vapor emitting regions for Class 0 , Class I, and Class II protostellar systems. A significant fraction of the water vapor resides in the warm inner envelope of Class 0 objects. Meanwhile, various physical and chemical models of Class II disks indicate three major water reservoirs from hot $\left(T_{\text {dust }}>160 \mathrm{~K}\right.$, region 1) to warm $\left(T_{\text {gas }}>T_{\text {dust }}\right.$, region 3$)$ to cold $\left(T_{\text {dust }}<20 \mathrm{~K}\right.$, region 2$)$. The most abundant water vapor is located in Region 1. From this work, the water vapor reservoir in Class I objects that can be probed with the $\mathrm{H}_{2}^{18} \mathrm{O}$ mm data is most likely similar to that of Class II disks that resides in the inner $10 \mathrm{au}$.

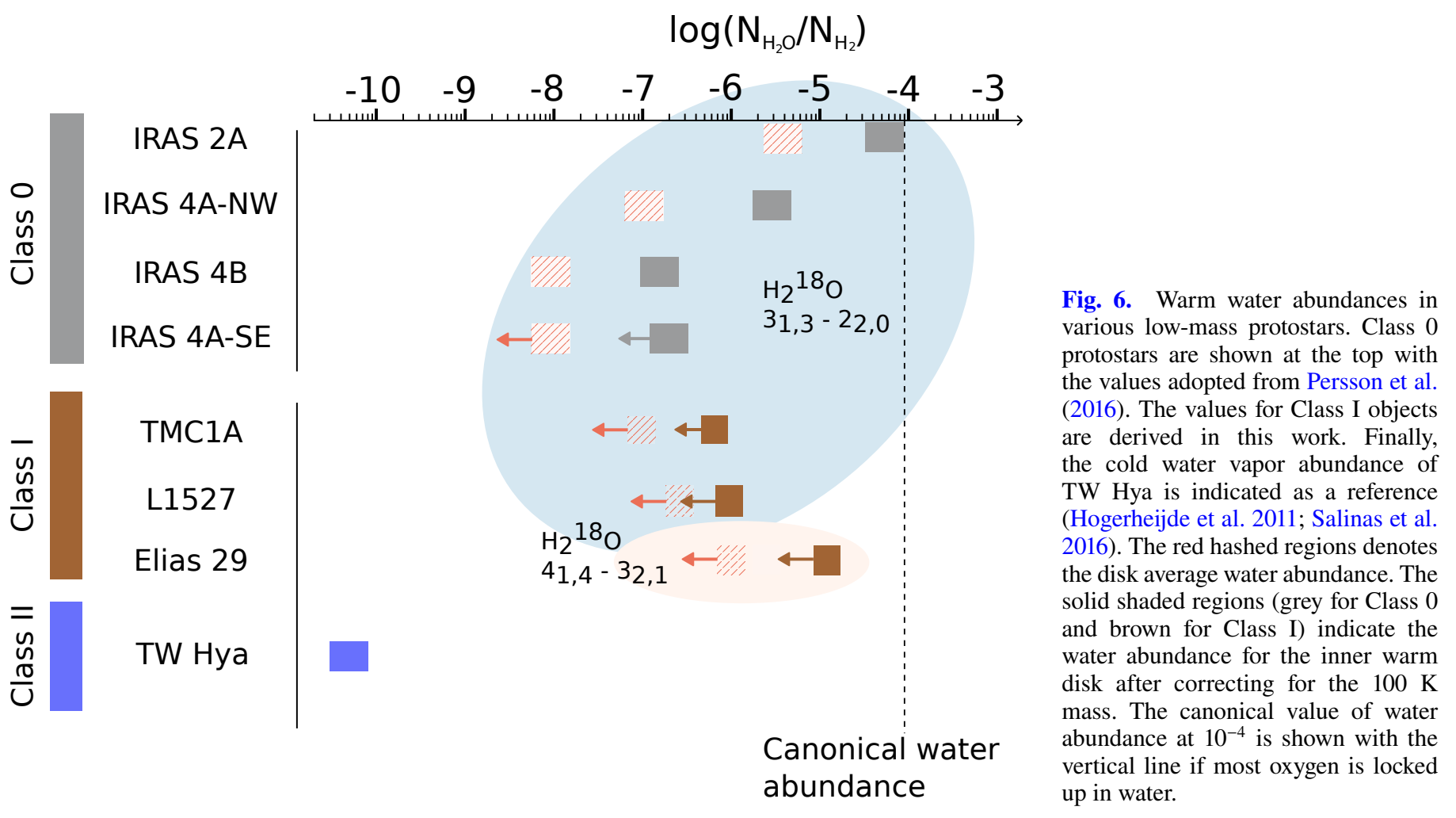

provide crucial implications on the physical and chemical structure of the inner warm regions of Class I protostars.

Figure 6 shows the water abundance averaged over the disk across the different stages of low-mass star formation. We include the cold water abundance of the TW Hya disk (Salinas et al. 2016) for comparison. We note that the cold water abundance traces the water reservoir that is released to the gas phase through a nonthermal mechanism (UV photodesorption,
Dominik et al. 2005; Hogerheijde et al. 2011; Salinas et al. 2016; Du et al. 2017), rather than thermal desorption. From the abundances, the maximum upper limits to the warm water abundances in Class I disks either averaged over the entire disk or dust temperatures $>100 \mathrm{~K}$ regions are closer to the abundances in Class 0 objects. On the other hand, the upper limits to the water vapor column densities in Class I disks are significantly lower than the water column densities in Class 0 disk-like structures 
(Table 4). Thus, in terms of the total amount of water vapor, Class I disks are clearly drier than Class 0 disk-like structures. More importantly, the envelope around Class I disks are too tenuous to emit observable $\mathrm{H}_{2}^{18} \mathrm{O}$ emission as shown in Fig. 4 as a result of low envelope mass and low water abundance on average over the inner 50 au diameter similar to that of the Class 0 studies.

\subsection{Water evolution during star and planet formation}

While the number of water detections toward protostellar disks is still low, we have a small sample that can be used to propose a water delivery mechanism during star and planet formation. Our underlying assumption is that the water vapor reservoir in Class I disks follows the standard picture of water reservoirs as outlined by the Class II disk studies (see Sect. 4.4). In addition, in the picture of disk formation (Hueso \& Guillot 2005; Visser et al. 2009), the water-rich icy dust grains are transported from the large-scale envelope to the outer disk unaltered with a water abundance of $\sim 10^{-4}$ with respect to $\mathrm{H}_{2}$. Once these dust grains cross the water iceline, the ices sublimate such that the water vapor abundance inside the water iceline is $10^{-4}$. Thus, the nondetections provide clues on how water is transported to planet-forming disks. Our upper limits suggest that the water abundance inferred through the millimeter water lines decreases as the disk forms and evolves ( $\lesssim 10^{5} \mathrm{yr}$, Visser et al. 2009). First, we present a few possible scenarios that can explain the nondetections of water emission in Class I disks.

Water vapor is expected to be abundant inside of the water iceline $\left(T_{\text {dust }}>100 \mathrm{~K}\right)$. We have shown in Sect. 4.3 and Table 3 that the overall water vapor abundance in the inner warm disk is still lower than this canonical value despite the fact that it is a factor of 10 higher than the disk-averaged value. To describe this region, we scaled the disk mass to obtain the $100 \mathrm{~K}$ mass adopting a power-law surface density profile $\Sigma \propto R^{-1}$. An alternative is to assume a steeper power-law slope $\left(\Sigma \propto R^{-1.75}\right)$ in order to avoid too many gravitationally unstable disks (Hartmann \& Bae 2018). A disk whose mass is distributed following a steeper slope has most of its mass in the inner few au. The consequence of a steeper power-law slope would be that the expected water column density inside 25 au radius would be higher than observed while the inferred average abundance would remain to be the same value. Thus, a steeper power-law profile is not the solution.

To simplify the analysis in order to compare with the Class 0 results, we have used the spherical envelope and disk models to estimate the extent of the $100 \mathrm{~K}$ region. The bolometric luminosities of the targeted Class I objects imply accretion rates between $10^{-9}$ and $10^{-6} M_{\odot} \mathrm{yr}^{-1}$ (see Ohashi et al. 1997b; Tobin et al. 2012; van 't Hoff et al. 2018). Based on the accretion rates, the midplane water iceline could extend to as far as $10 \mathrm{au}$. We now consider that the water snow surface is extended vertically from the midplane such that water vapor is abundant inside of $10 \mathrm{au}$. For a $0.01 M_{\odot}$ disk and a canonical water abundance, a water column density of at least $\sim 10^{19} \mathrm{~cm}^{-2}$ is available in the inner 10 au compared to $\sim 10^{18} \mathrm{~cm}^{-2}$ normalized over 25 au radius corresponding to an $\mathrm{H}_{2}^{18} \mathrm{O}$ column density of $\sim 10^{15} \mathrm{~cm}^{-2}$. With our observations, the $\mathrm{H}_{2}^{18} \mathrm{O}$ emission should have been detected at both 203 and $390 \mathrm{GHz}$ if the water line were optically thin. However, the optical depth of the $\mathrm{H}_{2}^{18} \mathrm{O} 390 \mathrm{GHz}$ line is $>1$ while it is $\sim 0.3$ for the $203 \mathrm{GHz}$ line for such a water column density, which results in peak temperatures of the line of $\sim 5 \mathrm{~K}$ in a $0 . ' 4$ beam (for $T_{\mathrm{ex}}=200 \mathrm{~K}$ ), which should have been detectable toward Elias $29\left(T_{\mathrm{rms}} \sim 1.6 \mathrm{~K}\right)$. Thus, if most of the water vapor is in the inner $10 \mathrm{au}$, our observations should have detected their emission toward TMC1A, L1527, and Elias 29. The nondetections can be caused by optically thick dust continuum affecting the strength of the water emission.

The dust continuum optical depth is interesting since it is directly linked to the dust mass absorption coefficient $\kappa_{v}$, the uncertainty in the disk mass and its distribution. For a few Class I sources, it is known that larger grains are present in the inner 1000 au (e.g., Melis et al. 2011; Miotello et al. 2014; Harsono et al. 2018). Large cm-size dust grains seem to be common in young protostellar systems (e.g., Jørgensen et al. 2009; Kwon et al. 2009; Testi et al. 2014; Tychoniec et al. 2018). Settled ice-covered large grains can explain the low cold water vapor abundance in the outer regions of Class II disks (Salinas et al. 2016; Krijt et al. 2016; Du et al. 2017). Furthermore, the presence of large dust grains results in higher $\kappa_{\mathrm{mm}}$ which means that the derived disk masses are lower limit. It is plausible that the presence of these large dust grains also affects the strength of the warm water emission from Class I disks.

To assess the influence of dust grains on water emission, we estimate the $\mathrm{H}_{2}^{18} \mathrm{O} 203 \mathrm{GHz}$ emission from a power-law disk model with small dust grains $\left(\kappa_{v}=0.7 \mathrm{~cm}^{2} \mathrm{~g}^{-1}\right.$ at $\left.203 \mathrm{GHz}\right)$ and large dust grains $\left(\kappa_{v}=2 \mathrm{~cm}^{2} \mathrm{~g}^{-1}\right)$. We only consider the $203 \mathrm{GHz}$ line in this analysis since it is less affected by optical depth effects (gas and dust). In other words, the suppression of the molecular gas emission is stronger for the $390 \mathrm{GHz}$ transition within the adopted formalism. For this exercise, a power-law disk that is described by surface density distribution of $\Sigma \propto R^{-1}$ and a temperature power-law of $T \propto R^{-0.4}$ is adopted. An inner radius of the dust disk is set at the dust sublimation temperature of $1500 \mathrm{~K}$ calculated using a photospheric temperature of $4000 \mathrm{~K}\left(L_{\star}=1 L_{\odot}\right)$ and an outer radius of $100 \mathrm{au}$. The total disk mass is set at $0.03 M_{\odot}$ with a gas-to-dust ratio of 100 . The water is assumed to be abundant $\left(10^{-4}\right.$ w.r.t. $\left.\mathrm{H}_{2}\right)$ where $T_{\text {dust }}>100 \mathrm{~K}$. Optically thick source functions are used to estimate the strength of the line and their emitting regions. In the left panel of Fig. 7, the predicted dust continuum and water intensities normalized (not convolved) over the beam along with their respective optical depths are plotted. The water line is easily optically thick inside of 10 au at the line center while the dust continuum is optically thick at $3 \mathrm{au}$. The water-emitting regions can be assessed by plotting the difference between the water and the dust intensities which is shown on the right panel of Fig. 7. It demonstrates how the water emitting region decreases if larger dust grains are present in the disk. As a result, a lower flux density per beam is emitted by the water vapor inside of $10 \mathrm{au}$. Since the water line is optically thick, it is beam diluted such that the peak emission in a channel is at most between 1 and $2 \sigma$ levels with the current sensitivity. Therefore, dust grain evolution in young disks provides the most plausible explanation for the weak water lines toward Class I disks. With these assumptions, the $\mathrm{H}_{2}^{18} \mathrm{O} 3_{1,3}-2_{2,0}$ should be detected with ALMA at a spatial resolution of 10 au $(\leqslant 0)^{\prime} 1$ at a distance of $<140 \mathrm{pc}$ ) and 2-3 times deeper observations $\left(\sigma \sim 1-5 \mathrm{mJy}^{-1}\right.$ beam $^{-1}$ at a $0.3 \mathrm{~km} \mathrm{~s}^{-1}$ channel).

For the models of Harsono et al. (2015), the water flux is estimated by calculating the water column density inside the water snowline $\left(N_{\mathrm{H}_{2}^{18} \mathrm{O}}=\frac{M_{\mathrm{H}_{2} \mathrm{O}}}{\pi R^{2}}\right)$ and normalized over $R=25$ au. We adopt the same optically thin limit method presented in Sect. 4.2. These methods are valid for unresolved molecular line emission. Our predicted flux density using this simple method is similar to the value obtained from the Bosman et al. (in prep.) model that includes a more detailed radiative transfer calculation. Our method overestimates the water flux in Class I disks in the high accretion case which is more representative of Class 0 

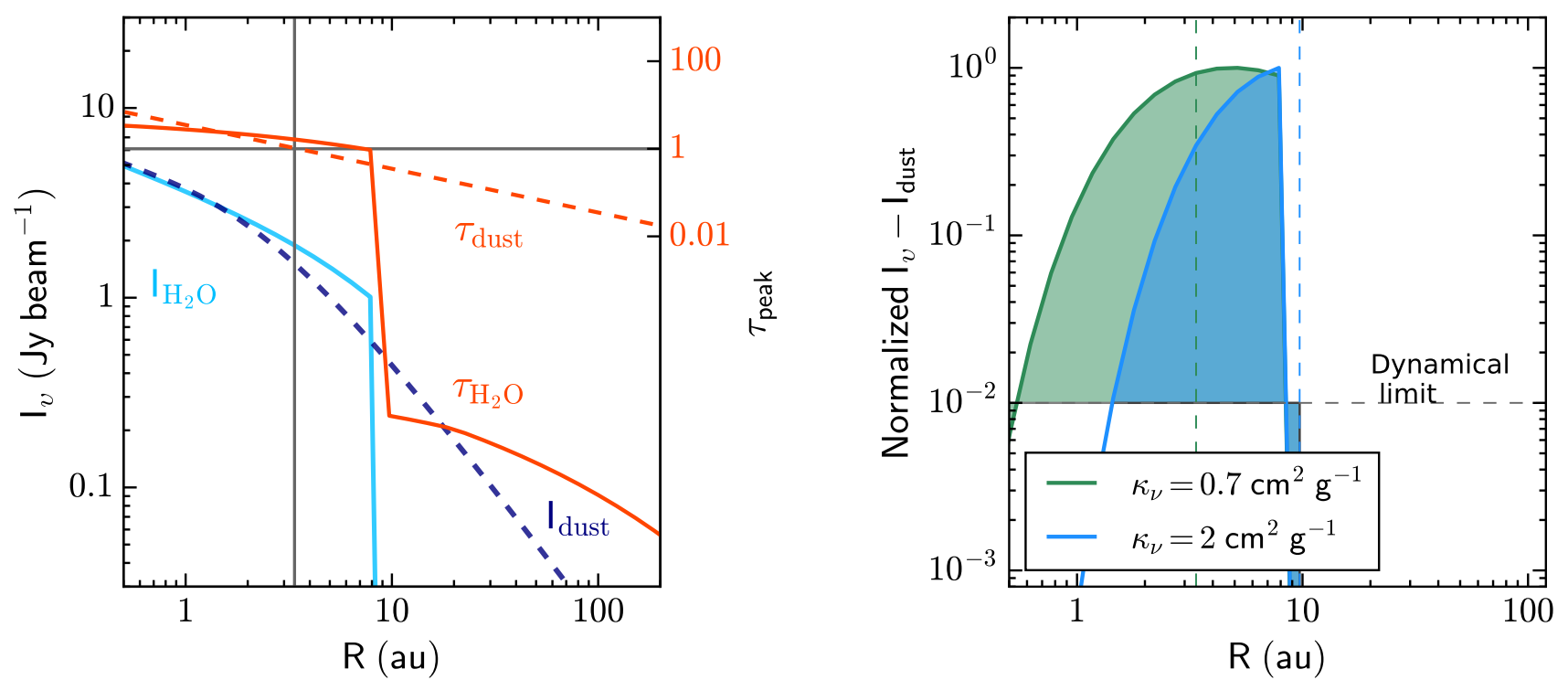

Fig. 7. Left: intensity profile normalized to 0 !'75 beam of the $\mathrm{H}_{2}^{18} \mathrm{O} 3_{1,3}-2_{2,0} 203 \mathrm{GHz}$ (light blue line) and dust continuum using $\kappa_{203 \mathrm{GHz}}=0.7 \mathrm{~cm}^{-2} \mathrm{~g}^{-1}$ (blue dashed line) for a power-law disk model with a water abundance of $10^{-4}$ with respect to $\mathrm{H}_{2}$ when $T_{\text {dust }}>100 \mathrm{~K}$ (see text). The optical depth of the water line and the dust are shown in solid and dashed red lines, respectively. Horizontal and vertical grey lines indicate the optical depth of one and its location, respectively. These intensity profiles have not been convolved with the synthesized beams. Right: normalized intensity profile including the dust attenuation and dust continuum subtraction. Two dust opacities are used to illustrate the influence of the dust mass absorption coefficient on the emergence of the water line. Vertical lines indicate the radii where the dust optical depth is larger than unity for $\kappa_{v}=0.7 \mathrm{~cm}^{2} \mathrm{~g}^{-1}$ (green) and $\kappa_{v}=2 \mathrm{~cm}^{2} \mathrm{~g}^{-1}$ (blue) at $203 \mathrm{GHz}$. Shaded regions show the simplified effective emitting region due to dynamical limit $(S / N \sim 30$ per velocity channel).

sources. These comparisons indicate that our observations are consistent with a (hidden) high water abundance $\left(10^{-4}\right)$ in the most inner warm disk ( $<10 \mathrm{au})$ while the envelopes around the Class I disks are dry on average.

Under the assumption that the inner warm disk should be abundant in water vapor, the results provide some hints on the water delivery during the early stages of star and planet formation. If the presence of large dust grains indeed suppresses the water emission in Class I disks, it implies that water is delivered to the young disk in the form of water ice locked by the settling of large dust grains since no water emission from any other disk or envelope reservoir is seen. Large dust grains have tendencies to form larger bodies that can lead to the formation of water-rich planetesimals (e.g., Raymond \& Izidoro 2017; Schoonenberg \& Ormel 2017). Such a large amount of water rich planetesimals implies an early delivery ofwater to Earth-like rocky bodies. The inner 10 au of these Class I disks should be abundant in water vapor as the small water-rich grains still drift inward and release the water vapor once the dust temperatures are above $100 \mathrm{~K}$ in these young disks. Alternatively, pressure bumps (Pinilla et al. 2012) could be present in these Class I disks preventing efficient drift of small dust grains to the inner warm disk. Deeper water observations at a higher-spatial resolution toward Class I disks are necessary to confirm the proposed early locking of volatiles during the star and planet formation. Based on these data, we propose that the majority of the ice-covered dust grains in prestellar cores to be transported to the planet-forming disks with little alteration.

\section{Summary and conclusions}

We present millimeter interferometric observations of water $\left(\mathrm{H}_{2}^{18} \mathrm{O}\right)$ toward five Class I protostellar objects (Elias 29, GSS30 IRS1, GSS30 IRS3, TMC1A, L1527). Our observations are sensitive to the Keplerian disks as revealed by the analysis of the dust continuum. In order to constrain the average water abundance, the $\mathrm{H}_{2}^{18} \mathrm{O}_{1,3}-2_{2,0}$ at $203 \mathrm{GHz}$ and $4_{1,4}-3_{2,1}$ at $390 \mathrm{GHz}$ lines are targeted to avoid the contamination by the outflow that is pervasive toward these embedded objects. The summary of the results are listed below.

- Dust continuum emission on small scales is detected toward Elias 29, GSS30 IRS1, and GSS30 IRS3 at $750 \mu \mathrm{m}$ with ALMA. NOEMA also detects the dust continuum emission toward TMC1A and L1527 at $203 \mathrm{GHz}$. Analysis of the continuum visibilities shows that our data are sensitive to the Keplerian disks around Elias 29, TMC1A and L1527. However, the nature of the compact disks around GSS30 IRS1 and GSS30 IRS3 is not constrained by our data.

- Neither NOEMA nor ALMA detects any water lines toward the targeted Class I disks. We report upper limits to the integrated water line intensities at scales of $100 \mathrm{au}$. The upper limits are extracted for the full extent of the Keplerian disk and inside of the water iceline $\left(T_{\text {dust }}>100 \mathrm{~K}\right)$ only. In the optically thin limit, the upper limits to the water vapor column densities are $<10^{18} \mathrm{~cm}^{-2}$ on scales of disks. These values are considerably lower than detected water column densities for Class 0 envelopes averaged over a projected 25 au radius. Thus, envelopes around Class I disks are drier based on the average water column density.

- Our upper limits to the water column density provide a stringent disk-averaged warm water abundance of $10^{-7}-10^{-6}$ with respect to $\mathrm{H}_{2}$ in Class I disks. By estimating the $T_{\mathrm{d}}>100 \mathrm{~K}$ mass with power-law disk models, the inferred water abundance is a factor of 10 higher with upper limits of $10^{-5}$ average over the inner warm disk. Our analysis suggests that the upper limits are still consistent with high water abundances in the inner warm disks around Class I objects ( $<10 \mathrm{au}$ ). Deep spatially resolved water observations toward these Class I disks are needed to confirm the presence of water vapor. 
- We have discussed the possible reasons for the nondetections of water emission in Class I disks. The most plausible and interesting scenario is that large millimeter and centimetersized dust grains are present in Class I disks. The presence of these dust grains suppresses the water emission from the inner $10 \mathrm{au}$. It also leads to optically thick water emission that is beam diluted by ourobservations such that the peak intensities of the water lines are below the current noise level. Based on the absence of any water vapor emission on scales larger than $10 \mathrm{au}$, we propose a scenario where water is delivered to the planet-forming disks by ice-covered large dust grains during disk formation.

Deep and high-spatial observations of water in both Bands 5 and 8 with ALMA toward Class 0 and I protostars are needed to place stronger constraints on the water evolution. In addition, both solid and vapor phases of water can be probed by future JWST observations that complement ground-based interferometric observations. Since the solid water feature is primarily seen for micron-size ice covered grains, millimeter ALMA observations are required to complete the picture of the early locking of volatiles in the early stages of planet formation during the formation of a disk.

Acknowledgements. This paper makes use of the following ALMA data: ADS/JAO.ALMA\#2013.1.00448.S. ALMA is a partnership of ESO (representing its member states), NSF (USA) and NINS (Japan), together with NRC (Canada) and NSC and ASIAA (Taiwan) and KASI (Republic of Korea), in cooperation with the Republic of Chile. The Joint ALMA Observatory is operated by ESO, AUI/NRAO and NAOJ. This work is based on observations carried out under project number X065 with the IRAM NOEMA Interferometer/PdBI. IRAM is supported by INSU/CNRS (France), MPG (Germany) and IGN (Spain). We are greatful to the IRAM staff, in particular to Tessel van der Laan, for preparing the observation and calibration of the data. We thank the anonymous referee for the constructive comments that improved the paper. Astrochemistry in Leiden is supported by the European Union A-ERC grant 291141 CHEMPLAN, by the Netherlands Research School for Astronomy (NOVA) and by a Royal Netherlands Academy of Arts and Sciences (KNAW) professor prize. J.C.M. acknowledges support from the European Research Council under the European Community's Horizon 2020 framework program (2014-2020) via the ERC Consolidator grant "From Cloud to Star Formation (CSF)" (project number 648505). M.V.P postdoctoral position is funded by the ERC consolidator grant 614264. This research made use of Astropy community-developed Python package for Astronomy (Astropy Collaboration 2018), numpy, matplotlib, and python package CASACORE to handle CASA products (images and measurement sets).

\section{References}

Andrews, S. M., Wilner, D. J., Hughes, A. M., Qi, C., \& Dullemond, C. P. 2009 ApJ, 700, 1502

Antonellini, S., Kamp, I., Riviere-Marichalar, P., et al. 2015, A\&A, 582, A105

Arasa, C., Koning, J., Kroes, G.-J., Walsh, C., \& van Dishoeck, E. F. 2015, A\&A, 575, A121

Artur de la Villarmois, E., Jørgensen, J. K., Kristensen, L. E., et al. 2019, A\&A, 626, A71

Aso, Y., Ohashi, N., Saigo, K., et al. 2015, ApJ, 812, 27

Aso, Y., Ohashi, N., Aikawa, Y., et al. 2017, ApJ, 849, 56

Astropy Collaboration (Price-Whelan, A. M., et al.) 2018, AJ, 156, 123

Beckwith, S. V. W., Sargent, A. I., Chini, R. S., \& Guesten, R. 1990, AJ, 99, 924

Bergin, E. A., \& van Dishoeck, E. F. 2012, Phil. Trans. R. Soc. London A Math. Phys. Eng. Sci., 370, 2778

Bergin, E. A., Hogerheijde, M. R., Brinch, C., et al. 2010, A\&A, 521, L33

Bethell, T., \& Bergin, E. 2009, Science, 326, 1675

Bitner, M. A., Richter, M. J., Lacy, J. H., et al. 2008, ApJ, 688, 1326

Bjerkeli, P., van der Wiel, M. H. D., Harsono, D., Ramsey, J. P., \& Jørgensen, J. K. 2016, Nature, 540, 406

Bontemps, S., André, P., Kaas, A. A., et al. 2001, A\&A, 372, 173

Boogert, A. C. A., Tielens, A. G. G. M., Ceccarelli, C., et al. 2000, A\&A, 360, 683

Boogert, A. C. A., Gerakines, P. A., \& Whittet, D. C. B. 2015, ARA\&A, 53, 541

Bruderer, S., van Dishoeck, E. F., Doty, S. D., \& Herczeg, G. J. 2012, A\&A, 541, A91

Bruderer, S., Harsono, D., \& van Dishoeck, E. F. 2015, A\&A, 575, A94
Burke, D. J., \& Brown, W. A. 2010, Phys. Chem. Chem. Phys., 12, 5947 Carney, M. T., Hogerheijde, M. R., Guzmán, V. V., et al. 2019, A\&A, 623, A 124

Carr, J. S., \& Najita, J. R. 2008, Science, 319, 1504

Chen, H., Myers, P. C., Ladd, E. F., \& Wood, D. O. S. 1995, ApJ, 445, 377

Chyba, C. F., \& Hand, K. P. 2005, ARA\&A, 43, 31

Coutens, A., Jørgensen, J. K., Persson, M. V., et al. 2014, ApJ, 792, L5

Di Francesco, J., Johnstone, D., Kirk, H., MacKenzie, T., \& Ledwosinska, E. 2008, ApJS, 175, 277

Dominik, C., Ceccarelli, C., Hollenbach, D., \& Kaufman, M. 2005, ApJ, 635, L85

Du, F., Bergin, E. A., Hogerheijde, M., et al. 2017, ApJ, 842, 98

Dunham, M. M., Vorobyov, E. I., \& Arce, H. G. 2014, MNRAS, 444, 887

Elias, J. H. 1978, ApJ, 224, 857

Endres, C. P., Schlemmer, S., Schilke, P., Stutzki, J., \& Müller, H. S. 2016, J. Mol. Spectr., 327, 95

Fedele, D., Bruderer, S., van Dishoeck, E. F., et al. 2013, A\&A, 559, A77

Friesen, R. K., Pon, A., Bourke, T. L., et al. 2018, ApJ, 869, 158

Gibb, E. L., Whittet, D. C. B., Boogert, A. C. A., \& Tielens, A. G. G. M. 2004, ApJS, 151, 35

Glassgold, A. E., Meijerink, R., \& Najita, J. R. 2009, ApJ, 701, 142

Goldsmith, P. F., \& Langer, W. D. 1978, ApJ, 222, 881

Goldsmith, P. F., \& Langer, W. D. 1999, ApJ, 517, 209

Gundlach, B., \& Blum, J. 2015, ApJ, 798, 34

Hall, C., Rice, K., Dipierro, G., et al. 2018, MNRAS, 477, 1004

Harsono, D., Alexander, R. D., \& Levin, Y. 2011, MNRAS, 413, 423

Harsono, D., Jørgensen, J. K., van Dishoeck, E. F., et al. 2014, A\&A, 562, A77

Harsono, D., Bruderer, S., \& van Dishoeck, E. F. 2015, A\&A, 582, A41

Harsono, D., Bjerkeli, P., van der Wiel, M. H. D., et al. 2018, Nat. Astron. 2, 646

Hartmann, L., \& Bae, J. 2018, MNRAS, 474, 88

Herczeg, G. J., Brown, J. M., van Dishoeck, E. F., \& Pontoppidan, K. M. 2011, A\&A, 533, A112

Hildebrand, R. H. 1983, QJRAS, 24, 267

Hogerheijde, M. R., \& Sandell, G. 2000, ApJ, 534, 880

Hogerheijde, M. R., Bergin, E. A., Brinch, C., et al. 2011, Science, 334, 338

Hueso, R., \& Guillot, T. 2005, A\&A, 442, 703

Ivezic, Z., \& Elitzur, M. 1997, MNRAS, 287, 799

Jacq, T., Henkel, C., Walmsley, C. M., Jewell, P. R., \& Baudry, A. 1988, A\&A, 199, L5

Jørgensen, J. K., \& van Dishoeck, E. F. 2010, ApJ, 710, L72

Jørgensen, J. K., van Dishoeck, E. F., Visser, R., et al. 2009, A\&A, 507, 861

Karska, A., Kaufman, M. J., Kristensen, L. E., et al. 2018, ApJS, 235, 30

Kauffmann, J., Bertoldi, F., Bourke, T. L., Evans, II, N. J., \& Lee, C. W. 2008, A\&A, 487, 993

Kitadai, N., \& Maruyama, S. 2018, Geosci. Front., 9, 1117

Kratter, K., \& Lodato, G. 2016, ARA\&A, 54, 271

Krijt, S., Ciesla, F. J., \& Bergin, E. A. 2016, ApJ, 833, 285

Kristensen, L. E., van Dishoeck, E. F., Bergin, E. A., et al. 2012, A\&A, 542, A8

Kristensen, L. E., Gusdorf, A., Mottram, J. C., et al. 2017a, A\&A, 601, L4

Kristensen, L. E., van Dishoeck, E. F., Mottram, J. C., et al. 2017b, A\&A, 605, A93

Kwon, W., Looney, L. W., Mundy, L. G., Chiang, H.-F., \& Kemball, A. J. 2009, ApJ, 696, 841

Lay, O. P., Carlstrom, J. E., \& Hills, R. E. 1997, ApJ, 489, 917

Lee, C.-F., Li, Z.-Y., \& Turner, N. J. 2020, Nat. Astron., 4, 142

Lommen, D., Jørgensen, J. K., van Dishoeck, E. F., \& Crapsi, A. 2008, A\&A, 481,141

Long, F., Herczeg, G. J., Pascucci, I., et al. 2017, ApJ, 844, 99

Loomis, R. A., Öberg, K. I., Andrews, S. M., et al. 2018, AJ, 155, 182

Mamajek, E. E. 2008, Astron. Nachr., 329, 10

McMullin, J. P., Waters, B., Schiebel, D., Young, W., \& Golap, K. 2007, ASP Conf. Ser., 376, 127

Meijerink, R., Pontoppidan, K. M., Blake, G. A., Poelman, D. R., \& Dullemond, C. P. 2009, ApJ, 704, 1471

Melis, C., Duchêne, G., Chomiuk, L., et al. 2011, ApJ, 739, L7

Melnick, G. J. 2009, ASP Conf. Ser., 417, 59

Miotello, A., Testi, L., Lodato, G., et al. 2014, A\&A, 567, A32

Motte, F., \& André, P. 2001, A\&A, 365, 440

Mottram, J. C., van Dishoeck, E. F., Schmalzl, M., et al. 2013, A\&A, 558, A126

Mottram, J. C., Kristensen, L. E., van Dishoeck, E. F., et al. 2014, A\&A, 572, A21

Mottram, J. C., van Dishoeck, E. F., Kristensen, L. E., et al. 2017, A\&A, 600, A99

Müller, H. S. P., Schlöder, F., Stutzki, J., \& Winnewisser, G. 2005, J. Mol. Struct., 742,215

Neufeld, D. A., Lepp, S., \& Melnick, G. J. 1995, ApJS, 100, 132 
Nisini, B., Giannini, T., \& Lorenzetti, D. 2002, ApJ, 574, 246

Notsu, S., Nomura, H., Ishimoto, D., et al. 2016, ApJ, 827, 113

Ohashi, N., Hayashi, M., Ho, P. T. P., et al. 1997a, ApJ, 488, 317

Ohashi, N., Hayashi, M., Ho, P. T. P., \& Momose, M. 1997b, ApJ, 475, 211

Olofsson, A. O. H., Olofsson, G., Hjalmarson, A., et al. 2003, A\&A, 402, L47

Ortiz-León, G. N., Loinard, L., Dzib, S. A., et al. 2018, ApJ, 869, L33

Ossenkopf, V., \& Henning, T. 1994, A\&A, 291, 943

Pérez, L. M., Carpenter, J. M., Andrews, S. M., et al. 2016, Science, 353, 1519

Persson, M. V., Jørgensen, J. K., \& van Dishoeck, E. F. 2012, A\&A, 541, A39

Persson, M. V., Jørgensen, J. K., van Dishoeck, E. F., \& Harsono, D. 2014, A\&A, 563, A74

Persson, M. V., Harsono, D., Tobin, J. J., et al. 2016, A\&A, 590, A33

Pinilla, P., Birnstiel, T., Ricci, L., et al. 2012, A\&A, 538, A114

Pontoppidan, K. M., Schöier, F. L., van Dishoeck, E. F., \& Dartois, E. 2002, A\&A, 393, 585

Raymond, S. N., \& Izidoro, A. 2017, Icarus, 297, 134

Robitaille, T. P., Whitney, B. A., Indebetouw, R., Wood, K., \& Denzmore, P. 2006, ApJS, 167, 256

Salinas, V. N., Hogerheijde, M. R., Bergin, E. A., et al. 2016, A\&A, 591, A122

Schmalzl, M., Visser, R., Walsh, C., et al. 2014, A\&A, 572, A81

Schoonenberg, D., \& Ormel, C. W. 2017, A\&A, 602, A21

Smith, R. G., Sellgren, K., \& Tokunaga, A. T. 1989, ApJ, 344, 413

Snell, R. L., Howe, J. E., Ashby, M. L. N., et al. 2000, ApJ, 539, L101

Stevenson, D. J., \& Lunine, J. I. 1988, Icarus, 75, 146

Tabone, B., Cabrit, S., Bianchi, E., et al. 2017, A\&A, 607, L6

Tafalla, M., Liseau, R., Nisini, B., et al. 2013, A\&A, 551, A116

Testi, L., Birnstiel, T., Ricci, L., et al. 2014, in Protostars and Planets VI, eds. H. Beuther, C. Dullemond, \& T. Henning (University of Arizona Press, Tucson), 339
Tobin, J. J., Hartmann, L., Chiang, H.-F., et al. 2012, Nature, 492, 83

Torres, R. M., Loinard, L., Mioduszewski, A. J., \& Rodríguez, L. F. 2009, ApJ, 698, 242

Tychoniec, Ł., Tobin, J. J., Karska, A., et al. 2018, ApJS, 238, 19

van der Tak, F. F. S., Walmsley, C. M., Herpin, F., \& Ceccarelli, C. 2006, A\&A, 447, 1011

van Dishoeck, E. F. 2004, ARA\&A, 42, 119

van Dishoeck, E. F., Herbst, E., \& Neufeld, D. A. 2013, Chem. Rev., 113, 9043

van Dishoeck, E. F., Bergin, E. A., Lis, D. C., \& Lunine, J. I. 2014, in Protostars and Planets VI, eds. H. Beuther, C. Dullemond, \& T. Henning (University of Arizona Press, Tucson), 835

van 't Hoff, M. L. R., Tobin, J. J., Harsono, D., \& van Dishoeck, E. F. 2018 A\&A, 615, A83

Visser, R., van Dishoeck, E. F., Doty, S. D., \& Dullemond, C. P. 2009, A\&A, 495, 881

Visser, R., Jørgensen, J. K., Kristensen, L. E., van Dishoeck, E. F., \& Bergin, E. A. 2013, ApJ, 769, 19

Vorobyov, E. I., \& Basu, S. 2005, ApJ, 633, L137

Walsh, C., Nomura, H., \& van Dishoeck, E. 2015, A\&A, 582, A88

Wang, K.-S., van der Tak, F. F. S., \& Hogerheijde, M. R. 2012, A\&A, 543, A22

Whittet, D. C. B., Bode, M. F., Longmore, A. J., et al. 1988, MNRAS, 233, 321

Wilson, T. L., \& Rood, R. 1994, ARA\&A, 32, 19

Woitke, P., Thi, W. F., Kamp, I., \& Hogerheijde, M. R. 2009, A\&A, 501, L5

Woitke, P., Pinte, C., Tilling, I., et al. 2010, MNRAS, 405, L26

Yen, H.-W., Koch, P. M., Takakuwa, S., et al. 2017, ApJ, 834, 178

Yıldız, U. A., Kristensen, L. E., van Dishoeck, E. F., et al. 2013, A\&A, 556, A89

Zhang, K., Pontoppidan, K. M., Salyk, C., \& Blake, G. A. 2013, ApJ, 766, 82 


\section{Appendix A: ALMA data: noise extractions and other spectral windows}
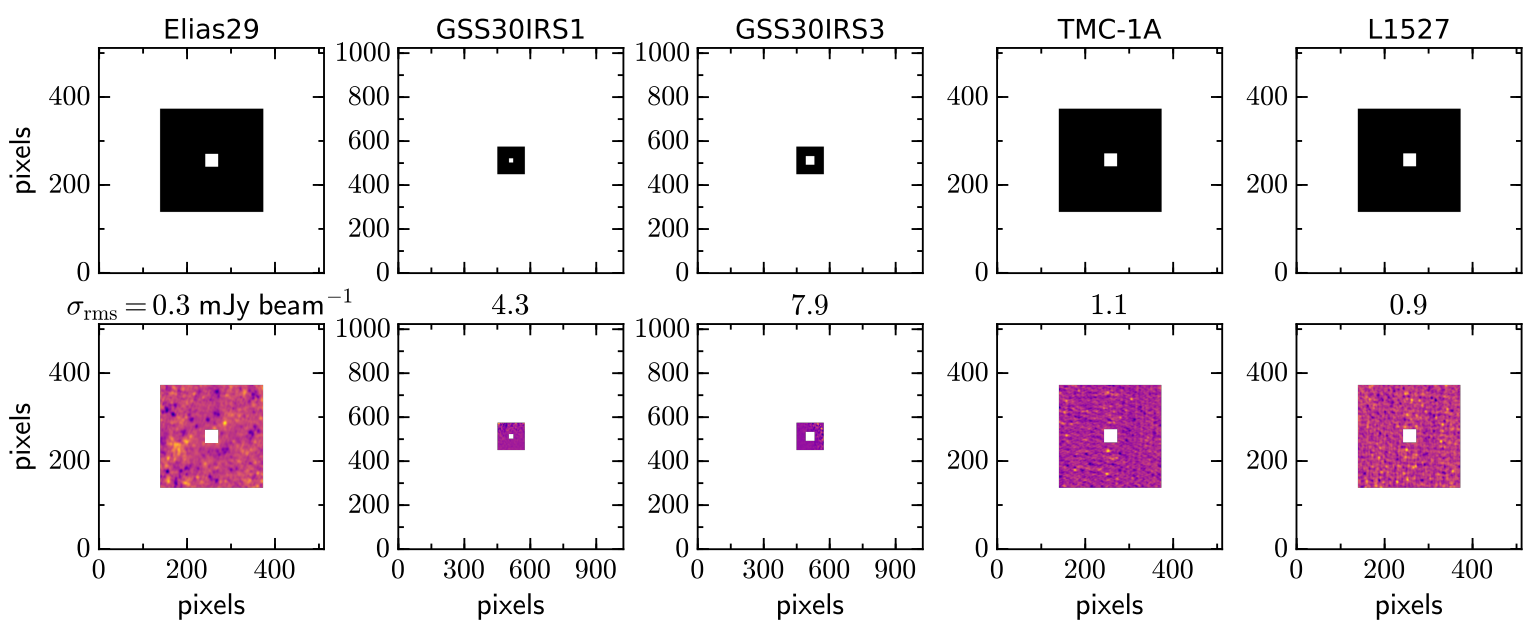

Fig. A.1. Top: masked continuum images where the black regions show the pixels that are used to calculate the noise level. Bottom: images after the masking showing that the source is not included with the noise level indicated in mJy per beam.

The noise levels of the images are extracted using square boxes as shown in Fig. A.1. A smaller extraction region is used for the GSS30 sources in order to obtain the appropriate higher noise levels at the edge of the primary beam. The continuum visibilities of the GSS30 regions are shown in Fig. A.2 whose amplitudes are much lower than the continuum images due to the incorrect phase centers. The low amplitudes are driven by the nonzero phases. Figure A.3 shows the spectra of other spectral windows with the identified transitions indicated. These spectra are taken by averaging over the dust continuum. These strong lines are due to $\mathrm{SiO}$ and $\mathrm{SO}_{2}$. The NOEMA spectra taken with the WideX and two low-spectral resolution windows are shown in Fig. A.4. For each target, there are four spectral windows: two widex windows and two continuum windows. For each spectral window, the spectrum average over the dust continuum emission and at the peak position are shown.
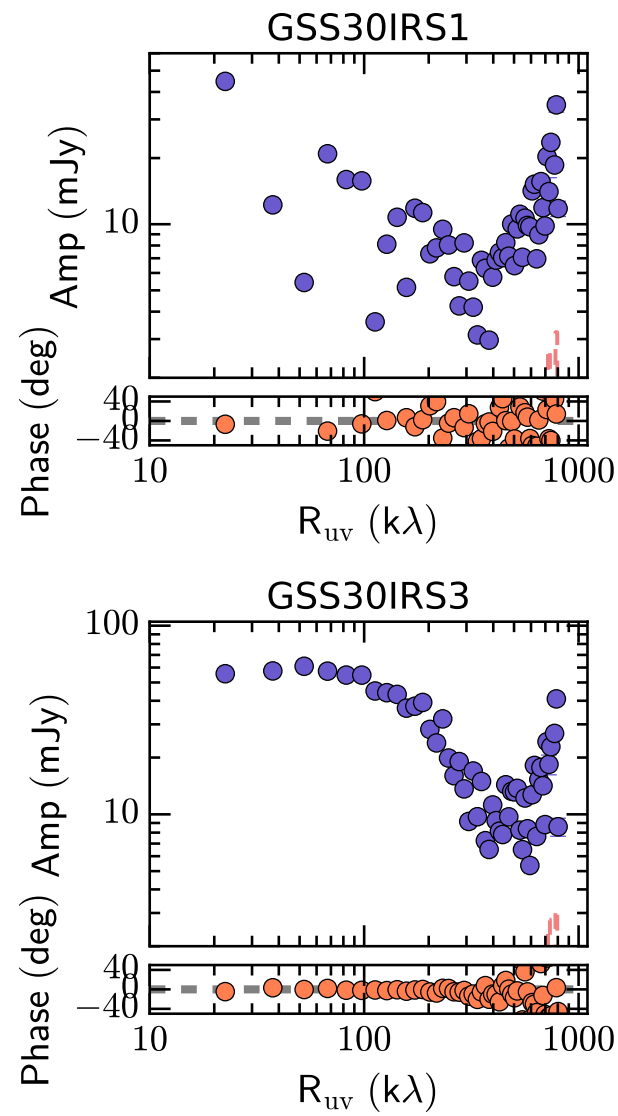

Fig. A.2. Circularly averaged binned amplitudes and phase as functions of projected baselines in $\mathrm{k} \lambda$ similar to Fig. 2. The visibilities for GSS30 are shown here in the Appendix due to the incorrect phase centers. 

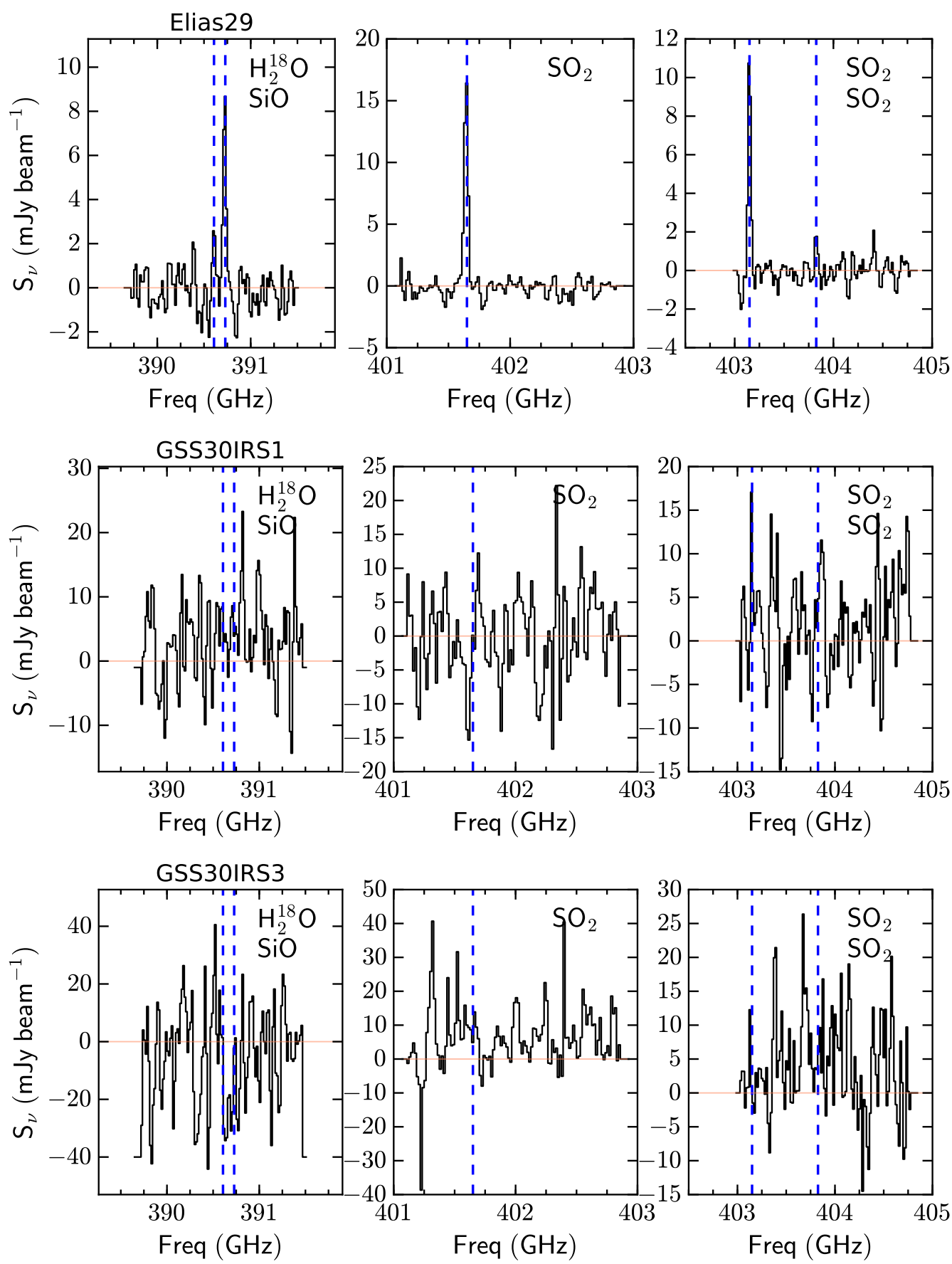

Fig. A.3. Spectra of the other spectral windows within our Band 8 ALMA data. Each spectrum is extracted by averaging over the pixels inside the dust continuum emission $>10 \sigma$ except for GSS30 IRS1 $(3 \sigma)$. The blue vertical dashed lines show some of the identified lines and the location of the $\mathrm{H}_{2}^{18} \mathrm{O} 4_{1,4}-3_{2,1} 390 \mathrm{GHz}$ as indicated. The identied molecules associated with the strong lines are indicated in the top right of each panel: top to bottom corresponds to left to right of the blue lines. 

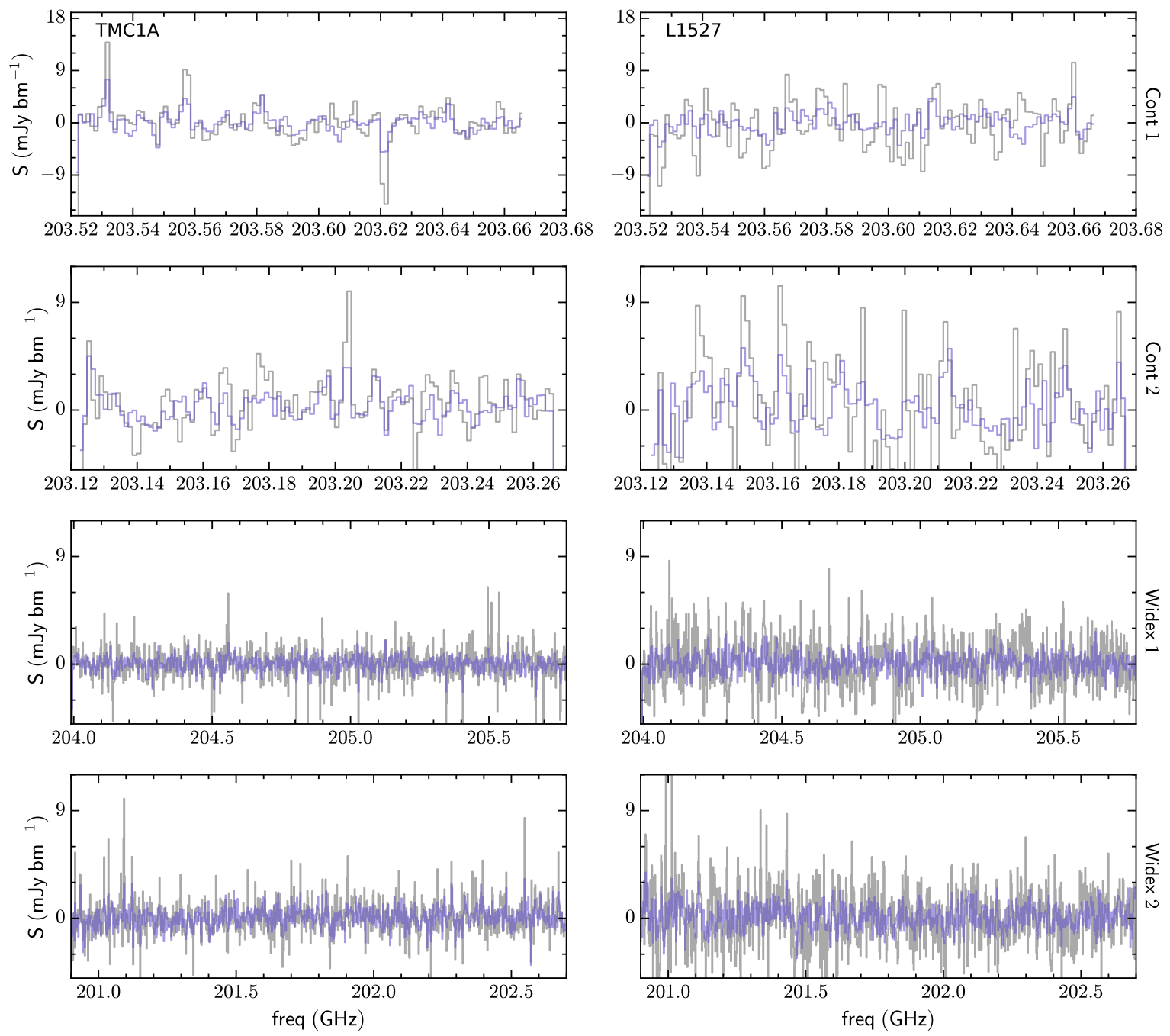

Fig. A.4. Spectra of the other spectral windows taken with the NOEMA observations at a lower spectral resolution. Some of the emissions are spurious signals due to clean artefacts. For each window, the spectrum is averaged over the dust continuum emission is shown in blue while the spectrum taken at the peak dust continuum position is shown in grey.

\section{Appendix B: Spherical envelope models}

A spherically symmetric power-law envelope $\left(n \propto r^{-p}\right)$ is considered with the dust temperature structure obtained through DUSTY (Ivezic \& Elitzur 1997). With a grid of models, Kristensen et al. (2012) fitted both the photometry at long wavelengths $(\lambda>100 \mu \mathrm{m})$ and the SCUBA images of four of our targets. By fitting the long wavelengths, the models place a constraint on the large-scale envelope structure $\left(r_{\text {out }}=y r_{\text {in }}\right)$. For completeness, the spherical envelope model parameters are tabulated in Table B.1. GSS30 IRS3 was not fitted in Kristensen et al. (2012) however this does not affect our results since we only considered the baselines $>200 \mathrm{k} \lambda$ to infer the disk structure. Figure B.1 shows the spectrum energy distribution of our Class I targets and the best-fit DUSTY models. The SCUBA 450 and 850 micron maps (Di Francesco et al. 2008) that were
Table B.1. DUSTY envelope parameters taken from Kristensen et al. (2012).

\begin{tabular}{clllll}
\hline \hline Source & $p$ & $y\left(\frac{r_{\text {out }}}{r_{\text {in }}}\right)$ & $\tau_{100}$ & $\begin{array}{l}r_{\text {in }} \\
(\mathrm{au})\end{array}$ & $\begin{array}{l}M_{\text {env }} \\
\left(M_{\odot}\right)\end{array}$ \\
\hline Elias 29 & 1.6 & 1000 & 0.1 & 15.5 & 0.04 \\
GSS30 IRS1 & 1.6 & 1000 & 0.2 & 16.2 & 0.1 \\
TMC1A & 1.6 & 900 & 0.4 & 7.7 & 0.2 \\
L1527 & 0.9 & 1200 & 0.3 & 5.4 & 0.9 \\
\hline
\end{tabular}

Notes. $\tau_{100}$ is the optical depth at $100 \mu \mathrm{m}$ that is used to attenuate the stellar spectrum.

used to constrain the extent of the protostellar envelopes are also presented. 

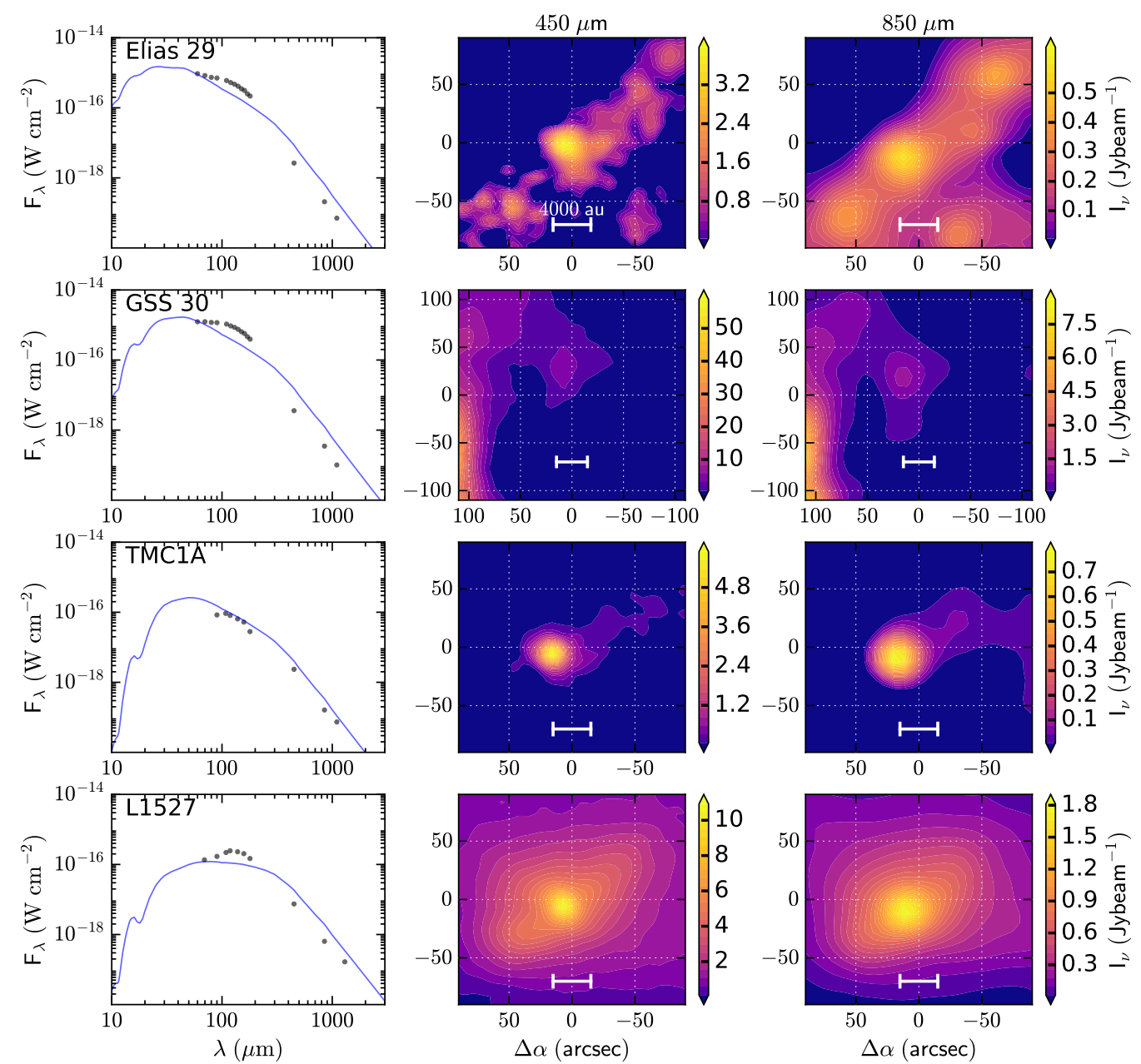

Fig. B.1. Observed spectral energy distribution (SED, left), SCUBA $450 \mu \mathrm{m}$ image (middle) and SCUBA $850 \mu \mathrm{m}$ image (right) for our Class I targets. The best-fit DUSTY model of Kristensen et al. (2012) produces the SED shown with the blue line. The color scale of the 450 and 850 micron maps extends from $10^{-2} \times$ peak intensity to peak intensity in 24 steps.

\section{Appendix C: Modeling of the disk structure}

The spherically symmetric envelope model used previously constrained envelope parameters from Kristensen et al. (2012, Appendix B) to create a dust continuum image with the radiative transfer code RATRAN (Hogerheijde \& Sandell 2000). The resulting image of the envelope is then mock-observed using the same setup as the observations (integration time, frequency, configuration, etc.) using either uvfmodel in GILDAS (for NOEMA observations) or fakeobs ${ }^{2}$ in CASA (for ALMA observations). The routines generate simulated continuum visibilities of the large-scale envelope that are subtracted from the continuum observations.

The remaining dust emission is assumed to be from a compact disk or a disk-like structure. The temperature of the disk is vertically isothermal and is described by a power-law profile $T \propto R^{-q}$ with a temperature of $1500 \mathrm{~K}$ at $0.1 \mathrm{au}$. Three different temperature power-law indices $q$ are used: $0.35,0.4$, and 0.5 . The resulting parameters are tabulated in Table C.1. The disk surface density follows a power-law distribution with radius following the formulas below

$$
\begin{aligned}
& \Sigma_{\text {disk }}(r)=\frac{\Sigma_{0}}{\Delta_{\mathrm{g} / \mathrm{d}}}\left(\frac{r}{r_{0}}\right)^{-p} \quad r<r_{\mathrm{c}}, \\
& \Sigma_{\text {taper }}(r)=\frac{\Sigma_{\mathrm{c}}}{\Delta_{\mathrm{g} / \mathrm{d}}} \exp \left[-\left(\frac{r}{r_{\mathrm{c}}}\right)^{2-p}\right] \quad r \geq r_{\mathrm{c}},
\end{aligned}
$$

where $\Sigma_{0}$ is the reference gas density at $r_{0}$, and a fixed gas-to-dust ratio $\Delta_{g / d}=100$. Beyond the disk (i.e. $r>r_{\mathrm{c}}$, where $\Sigma_{\mathrm{c}}=\Sigma_{\text {disk }}$ ) an exponential taper is applied for a smooth connection to the larger scales. To fit the parametrized disk, its orientation (inclination and position angle) has to be taken into account. To simplify and speed up the fitting we de-project the visibilities before fitting. The orientation is taken from the current best estimates, either from direct line observations of the rotating disk (Harsono et al. 2014) or assumed to be perpendicular to the outflow axis (Y1ldiz et al. 2013). Specific details of the disk model is given in Harsono et al. (2014) and Persson et al. (2016). The best-fit of parameters are determined by performing a greedy least square fit to the observed visibilities. 
D. Harsono et al.: Missing water in Class I disks

Table C.1. Best-fit parameters obtained from fitting a power-law disk to the continuum visibilities after subtracting the envelope's contribution.

\begin{tabular}{|c|c|c|c|c|c|c|}
\hline \multirow[b]{2}{*}{ Parameter } & \multicolumn{2}{|c|}{ NOEMA: $203 \mathrm{GHz}$} & \multicolumn{3}{|c|}{ ALMA Band 8: $390 \mathrm{GHz}$} & \\
\hline & TMC1A & L1527 & Elias29 ${ }^{(a)}$ & GSS30IRS $1^{(b)}$ & $\operatorname{GSS} 30 I R S 3^{(c)}$ & \\
\hline \multicolumn{7}{|c|}{$q=0.35$} \\
\hline$p$ & $1.2 \pm 0.2$ & $0.6 \pm 0.2$ & $0.8 \pm 0.2$ & $1 \pm 1$ & $0.7 \pm 0.2$ & \\
\hline$\Sigma_{50 \mathrm{au}}$ & $1.0 \pm 0.3$ & $2.7 \pm 0.4$ & $0.3 \pm 0.2$ & $0.1 \pm 0.4$ & $1 \pm 0.3$ & $\mathrm{~g} / \mathrm{cm}^{-2}$ \\
\hline$M_{\text {disk }}$ & $7 \pm 3$ & $23 \pm 5$ & $2.3 \pm 1.6$ & $0.7 \pm 3$ & $10 \pm 3$ & $10^{-3} \times M_{\odot}$ \\
\hline$M_{>100 \mathrm{~K}}$ & 2 & 3 & 0.4 & 0.2 & 2 & $10^{-3} \times M_{\odot}$ \\
\hline \multicolumn{7}{|c|}{$q=0.40$} \\
\hline$p$ & $1.2 \pm 0.2$ & $0.6 \pm 0.2$ & $0.8 \pm 0.2$ & $1 \pm 1$ & $0.7 \pm 0.2$ & \\
\hline$\Sigma_{50 \mathrm{au}}$ & $1.5 \pm 0.4$ & $3.9 \pm 0.6$ & $0.3 \pm 0.2$ & $0.2 \pm 0.05$ & $2 \pm 0.5$ & $\mathrm{~g} \mathrm{~cm}^{-2}$ \\
\hline$M_{\text {disk }}$ & $10 \pm 4$ & $34 \pm 7$ & $2.3 \pm 1.6$ & $1 \pm 4$ & $14 \pm 4$ & $10^{-3} \times M_{\odot}$ \\
\hline$M_{>100 \mathrm{~K}}$ & 5 & 12 & 1 & 0.7 & 2 & $10^{-3} \times M_{\odot}$ \\
\hline \multicolumn{7}{|c|}{$q=0.5$} \\
\hline$p$ & $1.3 \pm 0.2$ & $0.5 \pm 0.2$ & $0.9 \pm 0.3$ & $1 \pm 1$ & $0.6 \pm 0.2$ & \\
\hline$\Sigma_{50 \mathrm{au}}$ & $3.1 \pm 0.8$ & $11 \pm 2$ & $0.3 \pm 0.2$ & $0.3 \pm 0.1$ & $3 \pm 1$ & $\mathrm{~g} \mathrm{~cm}^{-2}$ \\
\hline$M_{\text {disk }}$ & $20 \pm 9$ & $100 \pm 20$ & $2.2 \pm 1.6$ & $2 \pm 7$ & $30 \pm 7$ & $10^{-3} \times M_{\odot}$ \\
\hline$M_{>100 \mathrm{~K}}$ & 7 & 12 & 0.5 & 0.5 & 4 & $10^{-3} \times M_{\odot}$ \\
\hline
\end{tabular}

Notes. The tabulated mass refers to the total mass of the disk using a gas-to-dust ratio of $100 .{ }^{(a)}$ Only $u-v$ distances between 200 and $730 k \lambda$ were fitted. ${ }^{(b)}$ Only $u-v$ distances between 200 and $800 k \lambda$ were fitted. Source located at edge of the primary beam. ${ }^{(c)}$ Only $u-v$ distances $>200 k \lambda$ were fitted. 